DOI 10.4171/JEMS/243

Artur Avila · Mikhail Lyubich · Weixiao Shen

\title{
Parapuzzle of the Multibrot set and typical dynamics of unimodal maps
}

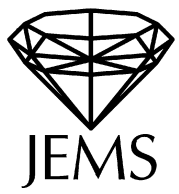

To the memory of Adrien Douady

Received July 3, 2008

Abstract. We study the parameter space of unicritical polynomials $f_{c}: z \mapsto z^{d}+c$. For complex parameters, we prove that for Lebesgue almost every $c$, the map $f_{c}$ is either hyperbolic or infinitely renormalizable. For real parameters, we prove that for Lebesgue almost every $c$, the map $f_{c}$ is either hyperbolic, or Collet-Eckmann, or infinitely renormalizable. These results are based on controlling the spacing between consecutive elements in the "principal nest" of parapuzzle pieces.

\section{Contents}

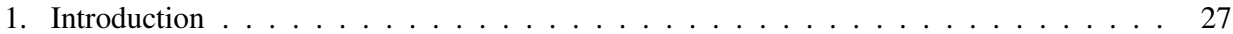

2. Holomorphic motions and a phase-parameter lemma . . . . . . . . . . . . . . 30

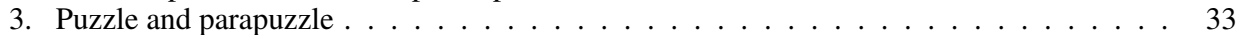

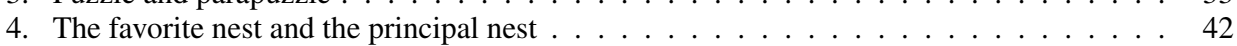

5. Slow recurrence $\ldots \ldots \ldots \ldots \ldots \ldots \ldots$

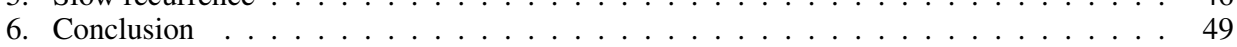

\section{Introduction}

In this paper we are concerned with the dynamics of unicritical polynomials

$$
f_{c}: z \mapsto z^{d}+c
$$

where $d \geq 2$, both on the real line (for real values of $c$ ) and on the complex plane (in the general case).

Until recently, the dynamical theory of the quadratic family $(d=2)$ was developed much deeper than its counterpart for the higher degree unicritical polynomials (see $[\mathrm{H}$, M2, L1, S], [L3]-[L5], [AM1]). The reason was that the quadratic maps possess some

A. Avila: CNRS UMR 7586, Institut de Mathématiques de Jussieu, 175 rue du Chevaleret, 75013 Paris, France; current address: IMPA, Estrada D. Castorina 110, Jardim Botânico, 22460-320 Rio de Janeiro, Brazil; e-mail: artur@math.sunysb.edu

M. Lyubich: Mathematics Department and IMS, Stony Brook University, Stony Brook, NY 11794, USA \& Department of Mathematics, University of Toronto; e-mail: mlyubich@ math.sunysb.edu

W. Shen: Mathematics Department, University of Science and Technology of China, Hefei, 230026, China; current address: Department of Mathematics, National University of Singapore, Block S17 (SOC1), 10 Lower Kent Ridge Road, Singapore 119076; e-mail: wxshen@ustc.edu.cn 
very special geometric features that distinguish them from their higher degree cousins. Recently, new tools have been developed [KL1, KL2, AKLS] that opened an opportunity to bring the higher degree case to the same level of maturity as the quadratic one ${ }^{1}$ In this paper that deals with the at most finitely renormalizable case, combined with forthcoming notes (see $[\mathrm{AL}]$ ) dealing with the infinitely renormalizable case, we intend to accomplish this goal.

For $d \geq 2$ fixed, let $\mathcal{M}=\mathcal{M}_{d}=\left\{c \in \mathbb{C}\right.$ : the Julia set of $f_{c}$ is connected $\}$ be the corresponding Multibrot set. The dynamics when $c \notin \mathcal{M}$ is always trivial, so we are mostly concerned with the description of the dynamics for $c \in \mathcal{M}$. When $d$ is odd, the real dynamics is trivial for all $c \in \mathbb{R}$, since $f_{c}$ is a homeomorphism, so when discussing real dynamics we will always assume that $d$ is even. In this case, for $c \in \mathcal{M} \cap \mathbb{R}, f_{c}$ is a unimodal map.

In what follows, various properties of a map $f_{c}$ will also be attributed to the corresponding parameter $c$. For a real $c$, the map $f_{c}$ (and the parameter $c$ itself) are called

- regular if $f_{c}$ has an attracting periodic orbit;

- infinitely renormalizable if there exist periodic intervals of an arbitrarily large (minimal) period;

- Collet-Eckmann if there exist $C>0$ and $\lambda>1$ such that

$$
\left|D f^{n}(c)\right| \geq C \lambda^{n}, \quad n=0,1,2, \ldots
$$

Such a map has a unique absolutely continuous invariant measure with strong statistical properties.

We can now formulate our main result on the real dynamics:

Theorem 1.1. For almost every $c \in \mathcal{M}_{d} \cap \mathbb{R}$, the map $f_{c}$ is either regular, or ColletEckmann, or infinitely renormalizable.

Remark 1.1. In [MN], Martens and Nowicki described a bigger class of unimodal maps that have an absolutely continuous invariant measure. In [L4], it was proved that for almost every real $c \in \mathcal{M}_{2}$, the quadratic polynomial $f_{c}$ is either regular, or MartensNowicki, or infinitely renormalizable. The Martens-Nowicki property was then replaced in [AM1] with the much stronger Collet-Eckmann property, thus providing us with Theorem 1.1 in the quadratic case.

Remark 1.2. With Theorem 1.1 in hand, we can go further, in the same way as in the quadratic case, to show that the whole fine statistical description of the dynamics of real quadratic maps [AM1], [AM3] is valid in the higher degree case as well.

Remark 1.3. In the forthcoming notes, the above result will be complemented by showing that the set of infinitely renormalizable parameters in $\mathcal{M}_{d} \cap \mathbb{R}$ has zero Lebesgue measure. (In the quadratic case, this was proved in [L5].)

\footnotetext{
1 See also [Sm KSS, BSS2] for recent advances in the higher degree case that use different tools.
} 
To stress the difference between the quadratic and the higher degree cases, let us mention one consequence of Theorem 1.1. Recall that a wild attractor for a unimodal map is a measure-theoretic attractor (in the sense of Milnor [M1]) which is not a topological attractor. There are no wild attractors in the quadratic family [L2], but they do exist for a sufficiently high even criticality $d$ [BKNS]. Moreover, if $d$ is large enough, the set of parameters $c \in \mathcal{M}_{d} \cap \mathbb{R}$ for which the wild attractor exists contains a Cantor set.

Corollary 1.2. For any even criticality $d$, the set of parameters $c \in \mathcal{M}_{d} \cap \mathbb{R}$ for which the wild attractor exists has zero Lebesgue measure.

Remark 1.4. One can show (using the estimates of this paper) that for large even $d$, the set of parameters $c \in \mathcal{M}_{d} \cap \mathbb{R}$ for which the wild attractor exists has positive Hausdorff dimension.

Remark 1.5. In [BSS2], it was proved that for almost every $c \in \mathcal{M}_{d} \cap \mathbb{R}$, the map $f_{c}$ admits a physical measure ${ }^{2}$ which is either supported on an attracting periodic orbit, or absolutely continuous (but $f_{c}$ is not necessarily Collet-Eckmann), or is supported on a uniquely ergodic Cantor set coinciding with the postcritical set (this possibility contains strictly the infinitely renormalizable case and the case of wild attractors).

The set of non-regular, non-infinitely renormalizable real parameters does have positive Lebesgue measure $[\bar{J}],[\mathrm{BC}]$. The situation is quite different for complex parameters:

Theorem 1.3. For almost any $c \in \mathbb{C}$, the map $f_{c}: z \mapsto z^{d}+c$ is either hyperbolic or infinitely renormalizable.

This result was proved for quadratic maps by Shishikura (see a sketch in $[\mathbf{S}]$; see also [AM2] for a proof closer to this paper). We actually prove the following estimate:

Theorem 1.4. Let $f_{c}$ be a non-renormalizable map with all fixed points repelling $4^{3}$ Then $c$ is not a density point of $\mathcal{M}_{d}$.

Again, the last two results are more surprising in the case $d>2$ : while all the quadratic maps in question have the Julia set of zero area ([L1], [S] $)$, it is conceivable that there exist higher degree non-renormalizable unicritical polynomials with the Julia set of positive measure. So, in the quadratic case the phase-parameter dictionary works in the natural way: zero area of Julia sets of the class of maps under consideration translates into zero area of the corresponding set of parameters. On the other hand, in the higher degree case, the phase-parameter correspondence is more subtle.

An important special feature of the quadratic maps essentially exploited in the previous work is the decay of geometry of the principal nest (see [L3, L4, AM1]). In this paper we demonstrate that, though in the higher degree case this property fails in general, it is satisfied for almost all non-regular non-infinitely renormalizable parameters. This is the key to all of the above results.

\footnotetext{
2 A measure $\mu$ is called physical if the Birkhoff averages of Lebesgue almost all orbits converge to $\mu$.

3 The result still holds under the assumption that $f_{c}$ is not infinitely renormalizable, and has all periodic orbits repelling. The argument for this generalization (which is more subtle than the usual application of the renormalization operator) is indicated in Remark 6.1
} 


\subsection{Notations}

- $\mathbb{D}_{r}=\{z:|z|<r\}, \mathbb{D}=\mathbb{D}_{1}, \mathbb{T}=\partial \mathbb{D}$.

- If $S$ is a hyperbolic Riemann surface, let dist ${ }_{S}$ be the hyperbolic metric in $S$ (with the usual normalization, so that in the upper-half plane $\mathbb{H}$ we have $\operatorname{dist}_{\mathbb{H}}(i, a i)=|\log a|$, $a>0$ ). The diameter of a subset $X \subset S$ with respect to dist ${ }_{S}$ will be denoted $\operatorname{diam}_{S} X$.

- $K(f)$ is the filled Julia set of $f$.

- $J(f)=\partial K(f)$ is its Julia set.

- Dil $(h)$ stands for the dilatation of a quasiconformal map $h$.

- $\bmod (A)$ stands for the modulus of the annulus $A$.

- Pullbacks of an open topological disk $V$ under $f$ are connected components of $f^{-1}(V)$.

- Pullbacks of a closed disk $V$ are closures of pullbacks of int $V$.

\section{Holomorphic motions and a phase-parameter lemma}

Let $\Lambda \subset \mathbb{C}$ be a Jordan disk. A holomorphic motion over $\Lambda$ (with base point $\lambda_{0} \in \Lambda$ ) of some set $Z \subset \mathbb{C}$ is a family of injective maps $h_{\lambda}: Z \rightarrow \mathbb{C}, \lambda \in \Lambda$, such that for every $z \in Z$, the "trajectory" (or the "orbit") $\lambda \mapsto h_{\lambda}(z)$ is holomorphic in $\lambda$ and $h_{\lambda_{0}}(z)=z$. Given such a holomorphic motion, we let $Z_{\lambda}=h_{\lambda}(Z)$.

The central result in the theory of holomorphic motions is the $\lambda$-lemma. It consists of two parts: extension and quasiconformality. The Extension Theorem (in its strongest version due to Słodkowski [S1]) says that a holomorphic motion $h_{\lambda}: Z \rightarrow \mathbb{C}$ over a Jordan disk $\Lambda$ can always be extended to a holomorphic motion $\hat{h}_{\lambda}: \mathbb{C} \rightarrow \mathbb{C}$ of the whole plane over the same $\Lambda$. The Quasiconformality Theorem (Mañé-Sad-Sullivan [MSS]) states that each $\hat{h}_{\lambda}$ is quasiconformal and

$$
\log \operatorname{Dil}\left(h_{\lambda}\right) \leq \operatorname{dist}_{\Lambda}\left(\lambda_{0}, \lambda\right) .
$$

We say that a holomorphic motion $h_{\lambda}: Z \rightarrow \mathbb{C}$ is continuous up to the boundary if the map $(\lambda, z) \mapsto h_{\lambda}(z)$ extends continuously to $\bar{\Lambda} \times Z$. A holomorphic motion $h_{\lambda}$ of a Jordan curve $T$ over $\Lambda$ which is continuous up to the boundary will be called a tubing of $T$ over $\Lambda$. Under these circumstances, a diagonal to the tubing is a holomorphic function $\psi$ in a neighborhood of $\bar{\Lambda}$ satisfying the following properties:

(D1) For $\lambda \in \Lambda, \psi(\lambda)$ belongs to the bounded component of $\mathbb{C} \backslash T_{\lambda}$, and for $\lambda \in \partial \Lambda$, $\psi(\lambda) \in T_{\lambda}$.

(D2) For any $\lambda \in \partial \Lambda$, the point $\psi(\lambda)$ has only one preimage $\gamma(\lambda) \in T$ under $h_{\lambda} \mid T$.

(D3) The holomorphic motion of a neighborhood of $\gamma(\lambda)$ in $T$ admits an extension over a neigborhood of $\lambda$.

(D4) The graph of $\psi$ crosses the orbit of $\gamma(\lambda)$ transversely at $\psi(\lambda)$.

(D5) The map $\gamma: \partial \Lambda \rightarrow T$ has degree 1.

Remark 2.1. Note that properties (D3) and (D4) imply that $\gamma: \partial \Lambda \rightarrow T$ is continuous, so that (D5) makes sense. 
Given a set $Z$ contained in the closed Jordan disk bounded by $T$, we say that a holomorphic (and continuous up to the boundary) motion $H_{\lambda}$ of $Z$ over $\Lambda$ fits the tubing of $T$ if for every $\lambda \in \bar{\Lambda}$, we have $H_{\lambda}(z)=h_{\lambda}(z)$ for $z \in Z \cap T$, while $H_{\lambda}(z) \notin h_{\lambda}(T)$ for $z \in Z \backslash T$.

Lemma 2.1. Let $h_{\lambda}: Z \rightarrow \mathbb{C}$ be a holomorphic motion over a Jordan disk $\Lambda$ continuous up to the boundary that fits a tubing of a Jordan curve T. Let $\psi$ be a diagonal to this tubing. Then for each point $z \in Z$ there exists a unique parameter $\lambda=\chi(z) \in \Lambda$ such that $h_{\lambda}(z)=\psi(\lambda)$. The map $\chi: Z \rightarrow \bar{\Lambda}$ is continuous and injective. Moreover, if $z \in \operatorname{int} Z$ and $h_{\chi(z)}$ is locally $K$-quasiconformal at $z$ then $\chi$ is locally $K$-quasiconformal at $z$.

Proof. Without loss of generality, we can assume that $\Lambda$ is the unit disk with base point at the origin. By assumptions (D3)-(D4), $\gamma: \partial \Lambda \rightarrow T$ is a local homeomorphism. By (D5), it has degree 1 , so that it is a homeomorphism. Letting $\chi \mid(Z \cap T)=\gamma^{-1}$, we see that the first assertion is valid for $z \in Z \cap T$.

Let $z \in Z \backslash T$. By applying an appropriate family of affine changes of variable, we can reduce the considerations to the case when $h_{\lambda}(z)=0, \lambda \in \overline{\mathbb{D}}$.

Let us consider a torus $\mathbb{T}_{0}^{2}=\mathbb{T} \times T \subset \mathbb{T} \times \mathbb{C}$. Let us deform it in $\mathbb{T} \times \mathbb{C}$ as follows:

$$
H_{r}: \mathbb{T}_{0}^{2} \rightarrow \mathbb{T}_{r}^{2},(\lambda, z) \mapsto\left(\lambda, h_{r \lambda}(z)\right), \quad \lambda \in \mathbb{T}, z \in T, 0 \leq r \leq 1 .
$$

Since the motion of the origin fits the tubing of $T$, the deformations never cross the core circle $\mathbb{T} \times\{0\}$.

Let us consider a family of curves $\psi_{r}: \mathbb{T} \rightarrow \mathbb{C}, \psi_{r}(\lambda)=h_{r \lambda} \circ \gamma(\lambda), 0 \leq r \leq 1$. Note that the graph of $\psi_{r}$ is a curve in $\mathbb{T}_{r}^{2}$ obtained by applying the homotopy $\bar{H}_{r}$ to the graph of $\psi_{0}$. Since the $\mathbb{T}_{r}^{2}$ are disjoint from the core circle, the curves $\psi_{r}$ never pass through the origin and hence have the same winding number around it. Since $\psi_{0}=\gamma$, by (D5) this winding number is equal to 1 . But $\psi_{1}=\psi \mid \mathbb{T}$ by definition of $\gamma$ (D2). By the Argument Principle, $\psi$ has a single root in $\mathbb{D}$, which proves the first assertion.

Any point $\lambda \in \Lambda$ has at most one preimage under $\chi$ since the maps $h_{\lambda}$ are injections. A point $\lambda \in \partial \Lambda$ has only one preimage $\chi^{-1}(\lambda)=\gamma(\lambda)$ by (D2) and the assumption that the motion of $Z$ fits the tubing of $T$. The graph of $\chi$ is the set of solutions $(z, \lambda)$ of $h_{\lambda}(z)=\psi(\lambda)$, which is clearly closed in $Z \times \bar{\Lambda}$, so $\chi$ is continuous.

Local quasiconformality of $\chi$ follows from the $\lambda$-lemma (see Corollary 2.1 of [L4]).

We will often encounter the situation when $Z$ contains an annulus $A$, and we want to obtain a lower bound on $\bmod (\chi(A))$. A trivial bound

$$
\bmod (\chi(A)) \geq K^{-1} \bmod (A), \quad \text { where } \quad K=\sup _{\lambda \in \Lambda} \operatorname{Dil}\left(h_{\lambda} \mid A\right),
$$

will sometimes be sufficient. However, since the dilatation of the holomorphic motion can blow up as $\lambda \rightarrow \partial \Lambda$, it will not cover all of our needs. Then we will make use of the following generalization of Corollary 4.5 of [L4]. 
Lemma 2.2. Under the above circumstances, let $X \subset Z \backslash T$, let $U_{\lambda}$ be the bounded component of $\mathbb{C} \backslash T_{\lambda}$, and let $U=U_{\lambda_{0}}$. Then:

(1) There exists a $\delta_{0}>0$ such that if $\operatorname{diam}_{U_{\lambda}} X_{\lambda}<\delta \leq \delta_{0}$ for every $\lambda \in \Lambda$ then $\operatorname{diam}_{\Lambda} \chi(X)<\epsilon(\delta), 4$ where $\epsilon(\delta) \rightarrow 0$ as $\delta \rightarrow 0$.

(2) Assume that $X$ is connected and $\operatorname{diam}_{U} X \leq M$. Assume also that for some $K>1$ and for every $\lambda \in \Lambda$, the map $h_{\lambda}: X \rightarrow \mathbb{C}$ extends to a $K$-qc homeomorphism $U \rightarrow U_{\lambda}$. Then $\operatorname{diam}_{\Lambda} \chi(X) \leq C=C(M, K)$.

Proof. (1) It is enough to consider the case where $X$ consists of two points. By a holomorphic change of coordinates $(\lambda, z) \mapsto\left(\lambda, \phi_{\lambda}(z)\right)$ where $\phi_{\lambda}: \mathbb{C} \rightarrow \mathbb{C}$ is affine, we may assume that $X_{\lambda}=\{0,1\}$ for all $\lambda \in \Lambda$. If $\operatorname{diam}_{U_{\lambda}} X_{\lambda}$ is small, then $\mathbb{D}_{2 R} \subset U_{\lambda}$ for some large $R>1$ and all $\lambda \in \Lambda$. Let $\tilde{h}$ be the holomorphic motion of $T \cup \mathbb{D}_{R}$ obtained by setting $\tilde{h}_{\lambda}(z)=h_{\lambda}(z)$ for $z \in T$ and $\tilde{h}_{\lambda}(z)=z$ for $z \in \mathbb{D}_{R}$. Notice that $\tilde{h}$ also fits the tubing and $\tilde{\chi}(X)=\chi(X)$.

Since $\tilde{h}$ is holomorphic in $\mathbb{D}_{R}, \tilde{\chi}=\psi^{-1}: \tilde{W} \rightarrow \Lambda$ is also holomorphic on $\mathbb{D}_{R}$. Hence $\bmod \left(\tilde{\chi}\left(\mathbb{D}_{R} \backslash \mathbb{D}\right)\right)=\frac{1}{2 \pi} \log R$ and

$$
\operatorname{diam}_{\Lambda}(\chi(X)) \leq \operatorname{diam}_{\tilde{\chi}\left(\mathbb{D}_{R}\right)} \tilde{\chi}(X)=O(1 / R) .
$$

(2) We will use the uniform equicontinuity of $K$-qc maps with respect to the hyperbolic metric: For any $K$-qc map $\phi: S \rightarrow \tilde{S}$ between hyperbolic Riemann surfaces,

$$
\operatorname{dist}(x, y)<\eta \Rightarrow \operatorname{dist}(\phi(x), \phi(y))<\delta(K, \eta),
$$

where $\delta(K, \eta) \rightarrow 0$ as $\eta \rightarrow 0$.

Let us select $\eta=\eta(K)$ so that $\delta(K, \eta)<\delta_{0}$, where $\delta_{0}$ comes from the first statement. We can cover $X$ by $N=N(\eta, M)$ sets $X_{1}, \ldots, X_{N}$ of hyperbolic diameter in $U$ bounded by $\eta$. Then the first statement is applicable to each $X_{i}$, so that $\operatorname{diam}_{\Lambda} \chi\left(X_{i}\right)<\epsilon_{0}=\epsilon\left(\delta_{0}\right)$. Since $X$ is connected,

$$
\operatorname{diam}_{\Lambda} \chi(X) \leq \sum \operatorname{diam}_{\Lambda} \chi\left(X_{i}\right)<N \epsilon_{0},
$$

and we are done.

We will need one lemma on lifting of a holomorphic motion by a family of branched coverings.

Lemma 2.3. Let $h_{\lambda}: Z \rightarrow Z_{\lambda}$ be a holomorphic motion over a pointed disk $\left(\Lambda, \lambda_{0}\right)$, and let $f_{\lambda}: U_{\lambda}^{\prime} \rightarrow U_{\lambda}$ be a holomorphic family of branched coverings of degree $d$ such that $U_{\lambda} \supset Z_{\lambda} \sqrt{5}$ Let $\Omega \Subset \Lambda$ be an open Jordan disk containing $\lambda_{0}$ such that for $\lambda \in \Omega$, the sets $Z_{\lambda}$ do not contain the critical values of $f_{\lambda}$. Then $h_{\lambda}$ over $\Omega$ lifts by $f_{\lambda}$ to a holomorphic motion $h_{\lambda}^{\prime}$ continuous up to the boundary.

\footnotetext{
4 On the other hand, one can show that the statement is false for large $\delta$.

5 We assume (as part of the definition of a "holomorphic family") that $\bigcup U_{\lambda}$ and $\bigcup U_{\lambda}^{\prime}$ are open subsets of $\mathbb{C}^{2}$.
} 
Proof. Each orbit $Z(z)=\left\{\left(\lambda, h_{\lambda}(z)\right): \lambda \in \Lambda\right\} \subset \mathbb{C}^{2}$ of the motion $h_{\lambda}$ lifts to a variety $Y(z)=\left\{(\lambda, z):\left(\lambda, f_{\lambda}(z)\right) \in Z(z)\right\}$ which properly projects to $\Lambda$ with degree $d$. Since for $\lambda \in \Omega, Z_{\lambda}$ does not contain critical values of $f_{\lambda}$, these varieties are unbranched over $\Omega$ and hence form a holomorphic motion $h_{\lambda}^{\prime}: Y \rightarrow Y_{\lambda}$ over it. All we need to show is that it is continuous up to the boundary of $\Omega$.

It is enough to show that for any compact $K \subset Y$, the family $\left\{\lambda \mapsto h_{\lambda}^{\prime}(y)\right\}_{y \in K}$ is uniformly equicontinuous over $\Omega$. Let $y_{n} \in K, \sigma_{n} \subset \Omega, \sigma_{n}$ an arc of diameter at most $1 / n$, and let $B_{n}=\left\{h_{\lambda}^{\prime}\left(y_{n}\right): \lambda \in \sigma_{n}\right\}$. We must show that the diameter of $B_{n}$ shrinks to 0 . We may assume that $y_{n} \rightarrow y \in K$ and $\sigma_{n} \rightarrow \lambda \in \bar{\Omega}$ in the Hausdorff topology. Then, for any $\epsilon>0$, for large $n, B_{n}$ lies within the $\epsilon$-neighborhood of $f_{\lambda}^{-1}\left(h_{\lambda}\left(f_{\lambda_{0}}(y)\right)\right)$. Since $f_{\lambda}^{-1}\left(h_{\lambda}\left(f_{\lambda_{0}}(y)\right)\right)$ has at most $d$ elements and $B_{n}$ is connected, this implies that $B_{n}$ has diameter at most $2 d \epsilon$, as desired.

\section{Puzzle and parapuzzle}

\subsection{Parameter and dynamical Böttcher coordinates}

The basic dynamical theory of the unicritical family $z \mapsto z^{d}+c$ (see [Sc1]) is similar to the basic theory of the quadratic family (see [DH1, M3]). For further reference, we recall here the main objects of the theory and set up notations.

The (dynamical) Böttcher function $B_{c}$ conformally conjugates $f_{c}$ near $\infty$ to $z \mapsto z^{d}$. The Green function $G_{c}=\log \left|B_{c}\right|$ extends harmonically to $\mathbb{C} \backslash K\left(f_{c}\right)$. Its level sets $\left\{G_{c}=\xi\right\}$ are called (dynamical) equipotentials $\mathcal{E}_{\xi}^{\text {dyn }}=\mathcal{E}_{\xi}^{\text {dyn }}(c)$. They form an invariant foliation with singularities at the precritical points (at each singularity, the equipotential looks locally like the intersection of $d$ lines). Let

$$
\Delta_{c}=\left\{z: G_{c}(z)>G_{c}(0)\right\} .
$$

It is the maximal neighborhood of $\infty$ saturated by the equipotentials on which the foliation is non-singular.

The gradient lines of $G_{c}$ coming from infinity are called (dynamical) external rays. They form a foliation of $\mathbb{C} \backslash K(f)$ slit along the gradient lines emerging from the critical points of the Green function. The argument ("angle") of $B_{c}$ is constant on each ray. The ray of angle $\theta$ is denoted by $\mathcal{R}_{\theta}^{\text {dyn }}=\mathcal{R}_{\theta}^{\text {dyn }}(c)$.

If the Julia set of $f$ is connected, the Böttcher function extends analytically to the whole basin of infinity, $\mathbb{C} \backslash K\left(f_{c}\right)$, and maps it conformally onto $\mathbb{C} \backslash \overline{\mathbb{D}}$.

Otherwise, $B_{c}$ extends analytically to the domain $\Delta_{c}$, and maps it conformally onto $\mathbb{C} \backslash \mathbb{D}_{\rho(c)}$, where $\rho(c)=e^{G_{c}(0)}>1$. In this case, the function

$$
B_{\mathcal{M}}(c)=B_{c}(c)
$$

is well defined and is called the (parameter) Böttcher function. It provides us with the Riemann mapping $\mathbb{C} \backslash \mathcal{M} \rightarrow \mathbb{C} \backslash \overline{\mathbb{D}}$. This basic relation between the dynamical and parameter Böttcher coordinates/Riemann mappings is the foundation of the phase-parameter correspondences for the unicritical families of polynomials. 
The (parameter) equipotentials and external rays, $\mathcal{E}_{\xi}^{\mathrm{par}}$ and $\mathcal{R}_{\theta}^{\mathrm{par}}$, are the level sets and the gradient lines of the parameter Green function $G_{\mathcal{M}}(c)=\log \left|B_{\mathcal{M}}(c)\right|$. They form two (non-singular) orthogonal foliations on $\mathbb{C} \backslash \mathcal{M}$. By basic relation 3.1,

- $c \in \mathcal{E}_{\xi}^{\mathrm{par}}$ iff $c \in \mathcal{E}_{\xi}^{\mathrm{dyn}}(c)$;

- $c \in \mathcal{R}_{\theta}^{\text {par }}$ iff $c \in \mathcal{R}_{\theta}^{\text {dyn }}(c)$.

(In each line, the first and the last " $c$ " stand for the parameter, while the intermediate one stands for the critical value.)

Let

$$
\mathbf{F}=\left\{(c, z) \in \mathbb{C}^{2}: z \in \mathbb{C} \backslash K\left(f_{c}\right)\right\}, \quad \boldsymbol{\Delta}=\left\{(c, z) \in \mathbb{C}^{2}: z \in \Delta_{c}\right\} ;
$$

these are open sets in $\mathbb{C}^{2}$. Let us also consider the critical set

$$
\mathcal{C}_{-}=\left\{(c, z) \in \mathbf{F}: \exists n \geq 0, f_{c}^{n}(z)=0\right\} ;
$$

it is an analytic subvariety in $\mathbf{F}$. The Böttcher function

$$
\mathbf{B}: \Delta \rightarrow \mathbb{C} \backslash \overline{\mathbb{D}}, \quad(c, z) \mapsto B_{c}(z),
$$

is a local holomorphic submersion, so that its level sets form a holomorphic foliation of $\boldsymbol{\Delta}$. Moreover, this foliation is transverse to the vertical foliation of $\mathbb{C}^{2}$, and thus determines a local holomorphic motion near any point $(c, z) \in \boldsymbol{\Delta}$.

Pulling this foliation back by the fiberwise dynamics $\mathbf{f}:(c, z) \mapsto\left(c, f_{c} z\right)$, we obtain a holomorphic foliation on $\mathbf{F}$ with singularities on $\mathcal{C}_{-}$. It determines a local holomorphic motion near any point $(c, z) \in \mathbf{F} \backslash \mathcal{C}_{-}$that we call the Böttcher motion.

We say that some holomorphic motion over parameter domain matches the Böttcher motion or respects the Böttcher coordinate if on the basin of infinity it coincides with the Böttcher motion. Such a motion preserves the external angles and heights of the points in the basin of infinity.

\subsection{Transversality to the diagonal}

Let $\mathcal{C}_{1}=\{(c, c): c \in \mathbb{C} \backslash \mathcal{M}\}$.

Lemma 3.1. Near any point $(c, c) \in \mathcal{C}_{1}$, the Böttcher motion is well defined and is transverse to $\mathcal{C}_{1}$.

Proof. The Böttcher motion is well defined since $\mathcal{C}_{1} \cap \mathcal{C}_{-}=\emptyset$. It is transverse to $\mathcal{C}_{1}$ since the Böttcher function $\mathbf{B} \mid \mathcal{C}_{1}$ is non-singular (as it conformally maps $\mathcal{C}_{1}$ onto $\mathbb{C} \backslash \mathcal{M}$ ).

Let $c_{0}$ be a Misiurewicz parameter, i.e., there is a repelling periodic point $a_{0}$, of period $q$, such that $f_{c_{0}}^{n}(0)=a_{0}$ for some $n \geq 1$, assumed to be minimal with this property. There are finitely many (and at least two) dynamical rays $\mathcal{R}_{\theta_{i}}^{\text {dyn }}\left(c_{0}\right)$ landing at $c_{0}$. Through a neighborhood of $c_{0}$, the Böttcher motion of these dynamical rays is well defined (see Lemma B.1 of $[\mathrm{GM}]$ and Lemma 2.2 of $[\overline{\mathrm{Sc} 1}])$. Their common landing point $p(c)$ is just the analytic continuation of $c_{0}$ as a preperiodic point (that is, $p(c)$ is the solution of $f_{c}^{n-1}(z)=f_{c}^{n-1+q}(z)$ near $\left.c_{0}\right)$. 
Lemma 3.2 (compare [vS]). Let $c_{0}$ be a Misiurewicz parameter as above. Then the curve $c \mapsto p(c)$ is transverse to the diagonal $c \mapsto c$ at $c_{0}$.

Proof. Let us consider one of the dynamical rays $\mathcal{R}_{\theta_{i}}^{\mathrm{dyn}}(c)$ landing at $p(c)$ which moves holomorphically under the Böttcher motion $h_{c}=B_{c}^{-1} \circ B_{c_{0}}$. By the basic dynamicalparameter relation, $\left\{c: c \in \mathcal{R}_{\theta_{i}}^{\mathrm{dyn}}(c)\right\}$ is a parameter ray $\mathcal{R}_{\theta_{i}}^{\text {par }}$ landing at $c$. Moreover, the map $\gamma: c \mapsto h_{c}^{-1}(c)$ is a homeomorphism from $\mathcal{R}_{\theta_{i}}^{\mathrm{par}}$ to $\mathcal{R}_{\theta_{i}}^{\mathrm{dyn}}\left(c_{0}\right)$. But if the curves $c \mapsto p(c)$ and $c \mapsto c$ had tangency of order $d \geq 1$ at $c_{0}$ then each point on $\mathcal{R}_{\theta}^{\text {dyn }}\left(c_{0}\right)$ would have $d+1$ preimages under $\gamma$ (compare Lemma 9.1 of [ALM] $)$ - contradiction.

\subsection{Parabolic wakes}

Let $\mathcal{A}$ stand for the set of parameters $c$ for which the map $f_{c}: z \mapsto z^{d}+c$ has an attracting fixed point $\alpha_{c}$. In the quadratic case, it is a domain bounded by the main cardioid of the Mandelbrot set. In the higher degree case, $\mathcal{A}$ is a domain bounded by a Jordan curve with $d-1$ cusps.

The set $\mathcal{M} \backslash \overline{\mathcal{A}}$ is disconnected. The closures of the connected components of $\mathcal{M} \backslash \overline{\mathcal{A}}$ are called (parabolic) limbs of $\mathcal{M}$. Each limb $\mathcal{L}$ intersects $\overline{\mathcal{A}}$ at a single point called the root $r=r_{\mathcal{L}}$ of the limb. The map $f_{r}$ has a parabolic fixed point with some multiplier $e^{2 \pi i \mathbf{p} / \mathbf{q}}$. There are two parameter external rays landing at the root. Their union with $r$ divides $\mathbb{C}$ into two (open) connected components: the one containing $\mathcal{L} \backslash\{r\}$ is called a parabolic wake $\mathcal{W}=\mathcal{W}_{\mathcal{L}}$ (see [DH1, $\left.\left.\left.\mathrm{M} 3\right], \mathrm{Sc1}\right]\right)$.

For $c \in \mathcal{L}$, the map $f_{c}$ has a unique dividing fixed point $\alpha_{c}$. There are $\mathbf{q}$ external rays $\mathcal{R}_{i}^{\text {dyn }}(c)$ landing at this point which are cyclically permuted by $f_{c}$ with combinatorial rotation number $\mathbf{p} / \mathbf{q}$. This configuration of $\mathbf{q}$ rays, together with the $\alpha$-fixed point, moves holomorphically over the whole parabolic wake $\mathcal{W}$. We let

$$
\Gamma^{0}=\Gamma^{0}(c)=\bigcup \mathcal{R}_{i}^{\mathrm{dyn}}(c) .
$$

Given some height $\xi>0$, let $\mathcal{W}(\xi)$ stand for the domain obtained by truncating the parabolic wake $\mathcal{W}$ by the parameter equipotential $\mathcal{E}_{\xi}^{\text {par }}$ of height $\xi$. For $c \in \mathcal{W}(d \xi)$, the Yoccoz puzzle pieces $Y_{j}^{0}=Y_{j}^{0}(c)$ of depth 0 are obtained by taking the closure of the connected components of $\mathbb{C} \backslash\left(\Gamma^{0}(c) \cup\left\{\alpha_{c}\right\}\right)$ truncated by the dynamical equipotential $\mathcal{E}_{\xi}^{\text {dyn }}(c)$ of height $\xi$ (where the piece containing 0 is also denoted $Y^{0}$ ). This configuration of $\mathbf{q}$ puzzle pieces moves holomorphically over $\mathcal{W}(d \xi)$.

Since $f_{c}(0) \notin \Gamma^{0}(c) \cup \mathcal{E}_{\xi}^{\text {dyn }}(c)$ for $c \in \mathcal{W}(\xi)$, the $f_{c}$-preimages of the rays $\Gamma^{0}(c)$ move holomorphically over $\mathcal{W}(\xi)$, and so do $f_{c}^{-1}\left(\mathcal{E}_{\xi}^{\mathrm{dyn}}(c)\right)=\mathcal{E}_{\xi / d}^{\mathrm{dyn}}$ and the $f_{c}$-preimages of $\alpha_{c}$. The closures of the components of $\mathbb{C} \backslash f_{c}^{-1}\left(\Gamma^{0}(c) \cup \alpha_{c}\right)$ truncated by the equipotential $\mathcal{E}_{\xi / d}^{\mathrm{dyn}}(c)$ are called Yoccoz puzzle pieces of depth 1 , and are denoted $Y_{j}^{1}$ (where the one containing 0 is also denoted $Y^{1}$ ).

We now fix some height $\xi$ (say, $\xi=1$ ): the moduli bounds in what follows will depend on this choice, but it will not be explicitly indicated. 


\subsection{Satellite copies of $\mathcal{M}$}

Let

$$
\mathcal{M}_{\mathcal{L}}=\left\{c \in \mathcal{L}: f^{\mathbf{q}}(0) \in Y^{1}, n=0,1,2, \ldots\right\} .
$$

This set is canonically homeomorphic to $\mathcal{M}$, and is called a satellite copy of the Multibrot set (see [DH2] $\backslash \mathrm{Sc2}]$ ). The maps $f_{c}$ with $c \in \mathcal{M}_{\mathcal{L}} \backslash\left\{r_{\mathcal{L}}\right\}$ (and the corresponding parameters $c$ ) are called satellite renormalizable.

\subsection{Decorations and Misiurewicz wakes}

Removing the satellite copy from the limb $\mathcal{L}$ disconnects it into countably many components, each attached to $\mathcal{M}_{\mathcal{L}}$ at a Misiurewicz parameter $c_{*}$ such that $f_{c_{*}}^{\mathbf{n q}}(0) \in f_{c_{*}}^{-1}(\alpha)$ $\backslash\{\alpha\}$ for some $\mathbf{n}>0$. The closures of these sets are called decorations.

There are $\mathbf{q}$ rays landing at $c_{*}$, dividing $\mathbb{C}$ into $\mathbf{q}-1$ Misiurewicz wakes and the component containing $\mathcal{M}_{\mathcal{L}} \backslash\left\{c_{*}\right\}$. The above number $\mathbf{n}$ is called the level of the Misiurewicz wake and the corresponding decoration.

For $c$ in the Misiurewicz wake, the level $\mathbf{n}$ is determined as the minimal natural number $n$ such that $f_{c}^{n \mathbf{q}}(0)$ belongs to some $Y_{j}^{1}(c) \neq Y^{1}(c)$. Let $\mathcal{O}_{k}^{\mathbf{n}}$ stand for the Misiurewicz wakes truncated by the parameter equipotential of height $\xi / d^{\mathbf{q}}$. Obviously, truncated Misiurewicz wakes are compactly contained in the corresponding truncated parabolic wake $\mathcal{W}(d \xi)$, and the Misiurewicz wakes attached to different roots have disjoint closures.

Define

$$
\Omega^{\mathbf{n}}=\mathcal{W}\left(\xi / d^{\mathbf{n q}-1}\right) \backslash \bigcup_{\mathbf{m}<\mathbf{n}} \bigcup_{k} \overline{\mathcal{O}}_{k}^{\mathbf{m}} .
$$

It is an open Jordan disk containing $\overline{\mathcal{O}}_{k}^{\mathbf{n}}$.

For the further understanding of the wakes, we need to go deeper into the puzzle.

The Yoccoz puzzle pieces of depth $n$ are the pullbacks of Yoccoz puzzle pieces of depth 0 under $f^{n}$. The puzzle pieces of depth $n$ will be denoted by $Y_{j}^{n}$, where the labels $j$ stand for the angles of the external rays that bound $Y_{j}^{n}$. They form a tiling of the neighborhood of $K(f)$ bounded by the equipotential of height $\xi / d^{n}$.

We also let $Y^{n}$ stand for the critical puzzle piece of depth $n$, i.e., $Y^{n} \ni 0$, while $Y_{v}^{n}$ stands for the puzzle piece containing the critical value.

We call $\bar{\Omega}^{\mathbf{n}}$ the parapuzzle piece of depth $\mathbf{n q}$, containing $\mathcal{M}_{\mathcal{L}}$. The closure of the Misiurewicz wake $\overline{\mathcal{O}}_{k}^{\mathbf{n}}$ will also be called a parapuzzle piece of depth $\mathbf{n q}+1$. We will now give a construction of the "parapuzzle pieces of depth $n \geq \mathbf{n q}+2$ " so that they will be the closures of the parameter domains over which the puzzle pieces of depth $n$ move holomorphically (with the same "combinatorics"). Moreover, they will form a tiling of the Misiurewicz wake, appropriately truncated.

Lemma 3.3. Fix some Misiurewicz wake $\mathcal{O}=\mathcal{O}_{k}^{\mathbf{n}} \subset \mathcal{W}$. Then all the boundaries of puzzle pieces up to depth $\mathbf{q n}$ move holomorphically over $\Omega^{\mathbf{n}}$, while the boundaries of puzzle pieces of depth $\mathbf{q n}+1$ move holomorphically over $\mathcal{O}$. All these motions provide us 
with tubings over $\mathcal{O}$ respecting the Böttcher coordinate. The critical value $c \mapsto c=f_{c}(0)$ is a diagonal of the tubing of $\partial Y_{v}^{\mathbf{q n}}$ over $\mathcal{O}^{\mathbf{n}}$. Moreover, for any $c \in \mathcal{O}$,

$$
\bmod \left(Y^{\mathbf{n q}}(c) \backslash Y^{\mathbf{n q}+1}(c)\right) \geq \delta(\mathcal{O})>0 .
$$

Proof. Since for $c \in \Omega^{\mathbf{n}}$, the critical orbit $f_{c}^{k}(0), k=0,1, \ldots, \mathbf{q n}$, does not cross the rays $\mathcal{R}_{i}^{\text {dyn }}(c)$ and the equipotential $\mathcal{E}_{\xi}^{\text {dyn }}(c)$, the configuration of Yoccoz puzzle pieces up to depth qn moves holomorphically over $\Omega^{\mathbf{n}} \ni \mathcal{O}$.

Similarly, for $c \in \mathcal{O}$, the critical value $f_{c}^{\mathbf{q n}+1}(0)$ does not cross the rays $\mathcal{R}_{i}^{\text {dyn }}(c)$ and the equipotential $\mathcal{E}_{\xi}^{\text {dyn }}(c)$ either, so that the puzzle pieces of depth qn +1 move holomorphically over $\mathcal{O}$. By Lemma 2.3 , this motion is continuous up to the boundary of $\mathcal{O}$.

We see that the boundary of each puzzle piece up to depth $\mathbf{q n}+1$ provides us with a tubing over $\mathcal{O}$. This tubing respects the Böttcher coordinate as it is induced by it.

Let us consider the puzzle piece $Y_{v}^{\mathbf{q n}}(c)$ moving holomorphically over $\Omega^{\mathbf{n}} \supset \mathcal{O}$ under the Böttcher motion $h_{c}$. It is bounded by two arcs of external rays with some angles $\theta_{+}$ and $\theta_{-}$(landing at the same point $a=a(c)$ such that $f^{\mathbf{q n}} a=\alpha$ ), and an arc of the equipotential $\mathcal{E}_{\xi / d \mathbf{q}^{\mathrm{n}}}^{\mathrm{dyn}}$. By the basic relation 3.1 , the Misiurewicz wake $\mathcal{O}$ is bounded by two arcs of external rays $\mathcal{R}_{\theta_{ \pm}}^{\text {par }}$ (landing at the Misiurewicz root $c_{*}$ such that $f_{c_{*}}^{\mathbf{q n}}\left(c_{*}\right)=\alpha$ ) and an arc of the equipotential $\mathcal{E}_{\xi / d}^{\mathrm{par}}$. Moreover, the parameter-phase map

$$
\gamma: \partial \mathcal{O} \rightarrow \partial Y_{v}^{\mathbf{q n}}, \quad c \mapsto h_{c}^{-1}(c),
$$

carries a parameter $c \in \partial \mathcal{O} \backslash\left\{c_{*}\right\}$ to the dynamical point $\gamma(c) \in Y_{v}^{\mathbf{q n}} \backslash\{a\}$ with the same Böttcher coordinates. This shows that the map $c \mapsto c$ satisfies properties (D1), (D2) and (D5) of the diagonal to the tubing of $\partial Y_{v}^{\mathbf{q n}}$ over $\mathcal{O}$. It satisfies (D3) and (D4) by the discussion in $\$ 3.2$.

Since the holomorphic motion $h$ over $\mathcal{O}$ extends to $\Omega^{\mathbf{n}}$,

$$
\bmod \left(Y^{0}(c) \backslash Y_{j}^{1}(c)\right) \geq \delta_{\mathbf{n}}>0, \quad c \in \mathcal{O},
$$

for any non-critical puzzle piece $Y_{j}^{1}$ contained in $Y^{0}$. Since

$$
f^{\mathbf{q n}}: Y^{\mathbf{q n}}(c) \backslash Y^{\mathbf{q n}+1}(c) \rightarrow Y^{0}(c) \backslash Y_{j}^{1}(c), \quad c \in \mathcal{O},
$$

is a covering of degree $d$ (for an appropriate non-critical puzzle piece $Y_{j}^{1}$ ), we obtain (3.2).

\subsection{Puzzle motion over the parapuzzle}

Let

$$
\Gamma^{n}=\Gamma^{n}(c)=f^{-n}\left(\Gamma^{0}\right) \backslash f^{-(n-1)}\left(\Gamma^{0}\right) .
$$

Thus, $\Gamma^{n}$ is the "new" ray boundary of the puzzle pieces of depth $n$ (which is not contained in the ray boundary of the puzzle pieces of depth $n-1$ ). 
We say that $f$ has well defined combinatorics up to depth $n$ if 0 belongs to the interior of a puzzle piece of depth $n$. Note that for $c \in \mathcal{W}\left(\xi / d^{\mathbf{q}}\right)$ with combinatorial rotation number $\mathbf{p} / \mathbf{q}$, combinatorics is well defined up to depth $\mathbf{q}+1$.

There are three ways the combinatorics can fail to be well defined at level $n$ :

- The Julia set $J(f)$ is connected and $f^{n}(0)=\alpha$. Such maps will be called $\alpha$-Misiurewicz.

- The Julia set is disconnected and the critical value $f^{n}(0)$ has height $\geq d \xi$. This situation will be essentially avoided by appropriate shrinking of the parameter domains.

- The Julia set $J\left(f_{c}\right)$ is disconnected and $f^{n}(0) \in \Gamma^{0}$. In this case, there are $d$ rays in $\Gamma^{n}$ that land at a precritical point. We call such precritical points pinching points of depth $n$ (if $n$ is the minimal integer with this property). Note that the pinching points of depth $n$ belong to the interior of the puzzle piece of depth $n-1$ (since by definition, they do not belong to $\left.f^{-(n-1)}\left(\Gamma^{0}\right)\right)$.

The combinatorics of $f$ up to depth $n$ (provided it is well defined) is the label of the puzzle piece of depth $n-1$ containing the critical value.

As we saw in $\$ 3.5$, all the maps $f_{c}, c \in \mathcal{O}$, have well defined combinatorics up to depth qn (and moreover, $\mathcal{O}$ is the maximal domain on which this is the case). We will now tile $\overline{\mathcal{O}}$ (truncated by appropriate equipotentials) according to the deeper combinatorics of the puzzle.

Lemma 3.4. The set of parameters $c \in \mathcal{O}$ with the same combinatorics $v$ up to depth $n \geq$ $\mathbf{q n}+1$ is an open Jordan disk bounded by the rays and equipotentials with the same angles and heights as the puzzle piece $Y_{v}^{n-1}$ containing the critical value. The closure of this disk, $\mathcal{Y}_{v}^{n}$, is called the parapuzzle of depth $n$ with combinatorics $v$. The boundaries of puzzle pieces of depth $n$ provide us with Böttcher tubings over $\mathcal{Y}_{v}^{n}$ that fit the tubings of the boundaries of puzzle pieces of depth $<n$ containing it. The critical value $\psi: c \mapsto$ $c=f_{c}(0), c \in \mathcal{Y}_{v}^{n}$, is a diagonal to the tubing of $\partial Y_{v}^{n-1}$. The parapuzzle pieces of depth $n$ tile the Misiurewicz wake $\mathcal{O}$ truncated by the equipotential of height $\xi / d^{n-1}$.

Proof. Assume inductively that the statement is true up to depth $n$ (where the base of induction is provided by the closed Misiurewicz wake $\overline{\mathcal{O}}=\mathcal{Y}^{\mathbf{q n}+1}$, see Lemma 3.3). Consider one of the parapuzzle pieces, $\mathcal{Y}=\mathcal{Y}_{j}^{n} \subset \overline{\mathcal{O}}$, and let us show how to tile its truncation by parapuzzle pieces of depth $n+1$.

Let us consider the boundary of a puzzle piece $X=Y_{k}^{n}$ contained in $Y_{v}^{n-1}$. By the induction assumption, it provides us with a tubing over $\mathcal{Y}$ that fits the tubing of $\partial Y_{v}^{n-1}$, and $\psi$ is a diagonal to the latter. Hence we can apply Lemma 2.1 and obtain an embedding $\chi: \partial X \rightarrow \mathcal{Y}$. The closed disk bounded by this Jordan curve is our parapuzzle $\mathcal{X}$ of depth $n+1$. Moreover, the map $\psi$ is the diagonal of the tubing of $\partial X$ over $\mathcal{X}$. Properties (D1), (D2) and (D5) of the diagonal follow directly from the construction, while properties (D3) and (D4) follow from the discussion of $\$ 3.2$.

Since $\partial X$ moves under the Böttcher motion and the diagonal $\psi$ is the identity $c \mapsto c$, the phase-parameter map $\chi: X \rightarrow \mathcal{X}$ respects the Böttcher coordinates. Hence the external angles and the heights of the rays and equipotentials forming $\partial \mathcal{X}$ are the same as those of $\partial X$. 
Let us now consider the puzzle pieces $Y_{k}^{n+1}$ of depth $n+1$. Since $f_{c}(0) \notin \partial Y_{j}^{n}(c)$ for $c \in \mathcal{X}$ and any $j$, these puzzle pieces move holomorphically over int $\mathcal{X}$ (obviously, respecting the Böttcher coordinates). This motion is continuous up to the boundary by Lemma 2.3 Let us show that it fits the tubing of the boundary of the puzzle piece of depth $n$ (and then inductively, of all smaller depths) containing it. Indeed, let $Y_{l}^{n+1} \subset Y_{k}^{n}$, and let $\zeta \in \partial Y_{l}^{n+1} \backslash \partial Y_{k}^{n}$. Then $\zeta \in \Gamma^{n+1}$, and since $\partial Y_{l}^{n+1}$ moves under the Böttcher motion, $h_{c}(\zeta) \in \Gamma^{n+1}(c)$ for any $c \in \mathcal{X}$. Hence $h_{c}(\zeta) \notin f_{c}^{-n}\left(\Gamma^{0}(c)\right)$, while the latter set contains $\partial Y_{k}^{n}(c)$. This provides us with the desired tubings of depth $n+1$ over $\mathcal{X}$.

Finally, the puzzle pieces $Y_{k}^{n}$ tile the puzzle piece $Y_{v}^{n-1}$ truncated by the equipotential of height $\xi / d^{n}$ and their simultaneous motion over $\mathcal{Y}$ fits the tubing of $\partial Y_{v}^{n-1}$. Applying Lemma 2.1 once again, we conclude that the corresponding parapuzzle pieces $\mathcal{X}=\mathcal{Y}_{k}^{n+1}$ tile the puzzle piece $\mathcal{Y}$ truncated by the equipotential of height $\xi / d^{n}$.

The parapuzzle piece of depth $n$ containing a point $c$ in its interior will also be denoted $\mathcal{Y}^{n}(c)$ (or $\mathcal{Y}^{n}$ when the choice of the base point $c$ is self-evident or non-essential). For instance, for $c \in \mathcal{O}=\mathcal{O}_{k}^{\mathbf{n}}$, we have

$$
\mathcal{Y}^{\mathbf{n q}}=\overline{\Omega^{\mathbf{n}}}, \quad \mathcal{Y}^{1+\mathbf{n q}}=\overline{\mathcal{O}_{k}^{\mathbf{n}}} .
$$

For $c \in \operatorname{int} \mathcal{Y}^{n}$, let $L_{n}=L_{n, c}: D_{n} \rightarrow Y^{n}$ be the first landing map to the critical puzzle piece $Y^{n}$. For $n \geq \mathbf{n q}+1$, its domain $D_{n}=D_{n}(c)$ consists of disjoint puzzle pieces $W_{i}^{n}=W_{i}^{n}(c)$ each of which is univalently mapped by $L_{n}$ onto $Y^{n}$. Note that $\mathbb{C} \backslash$ int $D_{n}$ is a forward invariant set, and int $D_{n}$ contains a dense subset of the filled-in Julia set $K(f)$.

Lemma 3.5. For $n \geq \mathbf{n q}+1$, the set $\mathbb{C} \backslash$ int $D_{n}$ moves holomorphically over int $\mathcal{Y}^{n}$. This motion is equivariant, i.e., $h_{c} \circ f_{c_{0}}=f_{c} \circ h_{c}$, and respects the Böttcher coordinate.

Proof. We fix some base parameter $c_{0} \in \mathcal{Y}^{n}$, and let $f=f_{c_{0}}, Y^{n}=Y^{n}\left(c_{0}\right)$, etc.

Let us first show that the boundary of each domain $W=W_{i}^{n}$ moves holomorphically over int $\mathcal{Y}^{n}$. Let $L_{n} \mid W=f^{l}$. Then $W$ has an itinerary $\left(\mu_{0}, \ldots, \mu_{l-1}, \mu_{l}=0\right)$, where $\mu_{m} \neq 0$ for $m<l$, satisfying the property

$$
f^{m}(W) \subset Y_{\mu_{m}}^{n}, \quad m=0, \ldots, l .
$$

For $c=c_{0}$, the restrictions $f \mid Y_{\mu_{m}}^{n}$ are univalent and

$$
f\left(Y_{\mu_{m}}^{n}\right) \supset Y_{\mu_{m+1}}^{n}, \quad m=0, \ldots, l-1 .
$$

Since the puzzle pieces $Y_{\mu}^{n}$ move holomorphically over int $\mathcal{Y}^{n}$, the same property is valid for all $c \in$ int $\mathcal{Y}^{n}$. Now, the repeated application of Lemma 2.3 shows that the boundaries of all $f^{m}(W), m=l-1, \ldots, 0$, move holomorphically over int $\mathcal{Y}^{n}$ as well.

By Lemma 3.4 for $c \in \operatorname{int} \mathcal{Y}^{n}, f_{c}(0) \in \operatorname{int} Y_{v}^{n-1}(c)$. Thus $0 \in \operatorname{int} Y^{n}(c) \subset \operatorname{int} D_{n}(c)$, and this implies that all precritical points are contained in int $D_{n}(c)$. Hence the Böttcher motion is well defined on $\mathbb{C} \backslash$ (int $D_{n} \cup K\left(f_{c}\right)$ ) (which is a dense subset of $\mathbb{C} \backslash$ int $D_{n}$ ). By 
the $\lambda$-lemma, this Böttcher motion extends to the whole set $\mathbb{C} \backslash$ int $D_{n}$, and this extension matches the previously constructed motion of $\bigcup_{i} \partial W_{i}^{n}$. The conclusion follows.

We let $h_{c}^{(n)}$ be the motion of $\mathbb{C} \backslash$ int $D_{n}$ over $\mathcal{Y}^{n}$ described in Lemma 3.5

We say that a puzzle piece $Y=Y_{\mu}^{k}\left(c_{0}\right)$ persists over depth $n$ if the boundary $\partial Y_{\mu}^{k}(c)$ moves holomorphically over int $\mathcal{Y}^{n}$ respecting the Böttcher coordinate. By Lemma 2.3 , if $n \geq 1+\mathbf{q n}$, this motion is continuous up to the boundary of $\mathcal{Y}^{n}$ and hence provides us with a tubing over $\mathcal{Y}^{n}=\mathcal{Y}^{n}\left(c_{0}\right)$. For instance, any puzzle piece $Y_{\mu}^{n}$ persists over depth $n$.

We say that a puzzle piece $Y_{\mu}^{k}$ is subordinate to depth $n$ if it is not properly contained in any domain $W_{i}^{n}$.

Lemma 3.6. Let $n \geq \mathbf{n q}+1$. If a puzzle piece $Y=Y_{\mu}^{k}$ is subordinate to depth $n$, then it persists over depth $n$. Moreover, its motion fits the tubing of the boundary of any bigger puzzle piece $Z=Y_{v}^{l}$ over $\mathcal{Y}^{n}$.

Proof. The first assertion follows from Lemma 3.5 since the boundary of $Y_{\mu}^{k}$ is contained in $\mathbb{C} \backslash$ int $D_{n}$.

Let us verify the second assertion. Since "fitting" is a transitive property, it is sufficient to check it for two consecutive depths, $l=k-1$. We may assume that $k>n$, since for $k \leq n$ the result follows from Lemma 3.4. Let us consider a puzzle piece $Y^{\prime}=f^{k-n}(Y)$ of depth $n$, and let $Z^{\prime}=f^{k-n}(Z)$. The latter is a puzzle piece of depth $n-1$ containing $Y^{\prime}$.

Let $h_{c}$ be the motion of $\mathbb{C} \backslash$ int $D_{n}$ from Lemma 3.5. Since it is equivariant up to the boundary of $\mathcal{Y}^{n}$, we have

$$
f_{c}^{k-n}\left(h_{c}(\partial Z)\right)=h_{c}\left(\partial Z^{\prime}\right), \quad c \in \mathcal{Y}^{n} .
$$

By Lemma 3.4, $\partial Y^{\prime}$ provides us with a tubing over $\mathcal{Y}^{n}$ that fits the tubing of $\partial Z^{\prime}$. By (3.4), this property lifts to imply that the tubing of $\partial Y$ fits the tubing of $\partial Z$. [Indeed, if $z \in \partial Y \cap$ int $Z$ then $f_{c_{0}}(z) \in \partial Y^{\prime} \cap$ int $Z^{\prime}$. Since the tubing of $\partial Y^{\prime}$ fits the tubing of $\partial Z^{\prime}$, $h_{c}\left(f_{c_{0}}^{k-n}(z)\right)=f_{c}^{k-n}\left(h_{c} z\right)$ does not belong to $h_{c}\left(\partial Z^{\prime}\right)$ for $c \in \mathcal{Y}^{n}$. By 3.4 , $h_{c} z$ does not belong to $h_{c}(\partial Z)$.]

A critical puzzle piece $Y^{n}$ is called a child of a critical puzzle piece $Y^{m}(m<n)$ if the map $f^{n-m}: Y^{n} \rightarrow Y^{m}$ is unicritical.

Corollary 3.7. Assume that for some $n \geq \mathbf{n q}+1$ and $k \geq 1$, the map $f^{k} \mid Y^{n}$ is unicritical (e.g., $Y^{n}$ is a child of some puzzle piece $Y^{m}$ and $\left.k \in[1, n-m]\right)$. Then the motion $h^{(n)}$ provides us with a tubing of $\partial f^{k}\left(Y^{n}\right)$ over $\mathcal{Y}^{n}$, and the critical value $c \mapsto f_{c}^{k}(0)$ is a diagonal to this tubing.

Proof. The first assertion follows from Lemma 3.6 since the piece $f^{k}\left(Y^{n}\right)$ is subordinate to depth $n$. The second assertion follows from Lemma 3.4 for $k=1$. Applying the family of univalent maps $f_{c}^{k-1}: f_{c}\left(Y^{n}(c)\right) \rightarrow f^{k}\left(Y^{n}(c)\right)$, we obtain it for any $k$.

If $f$ and $\tilde{f}$ have the same combinatorics up to depth $n$, a (Böttcher marked) pseudoconjugacy (up to depth $n$ ) between $f$ and $\tilde{f}$ is an orientation preserving homeomorphism $H:(\mathbb{C}, 0) \rightarrow(\mathbb{C}, 0)$ such that $H \circ f=\tilde{f} \circ H$ everywhere outside int $Y^{n}$, and which is the identity near infinity with respect to the Böttcher coordinates. 
Remark 3.1. By Lemmas 4.2 and 4.3 of [AKLS], if $c$ and $\tilde{c}$ have the same combinatorics up to depth $n$, and there exists a $K$-qc homeomorphism (int $\left.Y^{n}(c), 0\right) \rightarrow\left(\operatorname{int} Y^{n}(\tilde{c}), 0\right)$ which is the identity on the boundary with respect to the Böttcher coordinates, then $f_{c}$ and $f_{\tilde{c}}$ are $K$-qc pseudo-conjugate (up to depth $n$ ).

\subsection{Combinatorics of children}

If $f$ does not have well defined combinatorics of all depths, then either the Julia set of $f$ is disconnected or the critical point is eventually mapped to the repelling fixed point $\alpha$. Otherwise, we have critical puzzle pieces of all depths. In this case, we say that $f$ is combinatorially recurrent if the critical point returns to all critical puzzle pieces.

Given a critical puzzle piece $Y^{n}$, let $R_{Y^{n}}$ be the first return map to $Y^{n}$. The components of the domain of $R_{Y^{n}}$ are puzzle pieces, which are mapped by $R_{Y^{n}}$ onto $Y^{n}$, either univalently (if the component is non-critical), or $d$-to-1 (if the component is critical). Let $\mathrm{m}\left(Y^{n}\right)$ be the infimum, over all components $D$ of the domain of $R_{Y^{n}}$, of $\bmod \left(Y^{n} \backslash D\right)$.

If $f$ is combinatorially recurrent, then every critical puzzle piece has a child. These kids are ordered by "age": a child $Y^{k}$ is "older" than a child $Y^{l}$ if $Y^{k} \supset Y^{l}$ (and thus $k \leq l$ ). Note that the first child $Y^{k}$ of $Y^{n}$ coincides with the critical component of the domain of $R_{Y^{n}}$.

A combinatorially recurrent map is said to be primitively renormalizable if there exists a critical puzzle piece $Y^{n}$ such that the critical point never escapes its first child $Y^{k}$ under iterates of $R_{Y^{n}}:\left\{R_{Y^{n}}^{j}(0): j \geq 1\right\} \subset Y^{k}$. In general, we will say that a map $f$ is nonrenormalizable if it is neither satellite nor primitively renormalizable.

A child $Q=Y^{q}$ of $V=Y^{v}$ is called good if $f^{q-v}(0)$ is contained in the first child $U=Y^{u}$ of $V$. In this situation, $K=Y^{q-v+u}$ is a child of $U$ called the friend of $Q$. Note that $f^{q-v}: Q \backslash K \rightarrow V \backslash U$ is a covering map of degree $d$.

The favorite child of $V$ is the oldest good child $Q$ that appears after the first child $U$. One can see that the depth of the favorite child is the smallest integer $q>v$ such that $f^{q-v}(0)$ belongs to the first child $U$ and the orbit $\left\{f^{i}(0)\right\}_{i=1}^{q-v}$ passes through the annulus $V \backslash U$ (see the discussion preceding Lemma 2.3 of [AKLS]). If $f$ is combinatorially recurrent and non-renormalizable, then every critical puzzle piece has a favorite child.

\subsection{Phase-parameter transfer}

We will now apply Lemmas 2.1 and 2.2 to two dynamical situations that will often appear in what follows.

Lemma 3.8. Let us consider four levels $s<t \leq u<w$, where $u \geq \mathbf{n q}+1$, such that $Y^{u}$ is a child of $Y^{s}$ and $f^{u-s}\left(Y^{w}\right)$ is contained in some connected component $W=W_{i}^{t}$ of the first landing map to $Y^{t}$. Assume that

(1) $\bmod \left(Y^{s}(c) \backslash W(c)\right)>\delta$ for any $c \in \mathcal{Y}^{u}$;

(2) $f_{c}$ and $f_{\tilde{c}}$ are $K$-qc pseudo-conjugate up to depth $t$ for any $c, \tilde{c} \in \mathcal{Y}^{u}$.

Then $\bmod \left(\mathcal{Y}^{u} \backslash \mathcal{Y}^{w}\right)>\epsilon(K, \delta)$. 
Proof. Notice that $Y^{s}$ and $W$ are subordinate to depth $u$, and $c \mapsto f_{c}^{u-s}(0)$ is a diagonal to the tubing of $\partial Y^{s}$ over $\mathcal{Y}^{u}$. The first assumption implies that $\operatorname{diam}_{Y^{s}(c)} W(c)<$ $M=M(\delta)$ and the second assumption implies that the maps $h_{c}^{(t)} \mid\left(\partial Y^{s} \cup \partial W\right)$ have $K$-qc extensions to the whole complex plane, $c \in \mathcal{Y}^{u}$. Application of the second statement of Lemma 2.2 gives the result:

$$
\bmod \left(\mathcal{Y}^{u} \backslash \mathcal{Y}^{w}\right) \geq \bmod \left(\mathcal{Y}^{u} \backslash \mathcal{Y}^{\operatorname{depth}(W)+u-s}\right)>\epsilon(K, \delta) .
$$

Lemma 3.9. Let us consider four puzzle pieces $K \subset Q \subset U \Subset V$ of respective depths $k>q \geq u>v \geq \mathbf{n q}+1$. Assume that $U$ is the first child of $V, Q$ is a good child of $V$, and $K$ is the friend of $Q$. Let $\mathcal{K} \subset \mathcal{Q} \subset \mathcal{U} \Subset \mathcal{V}$ be the corresponding parapuzzle pieces. Then

$$
\bmod (\mathcal{Q} \backslash \mathcal{K})>\rho(\bmod (\mathcal{V} \backslash \mathcal{Q})) \bmod (V \backslash U),
$$

where $\rho: \mathbb{R}^{+} \rightarrow \mathbb{R}^{+}$is an increasing function.

Proof. Let $A=V \backslash U$. Since this annulus persists over $\mathcal{U}$, all the maps

$$
f_{c}: A \rightarrow f(A), \quad c \in \mathcal{U},
$$

are coverings of degree $d$.

By Corollary 3.7, the puzzle piece $f(U)$ (and of course, $f(V)$ ) persists over $\mathcal{V}$, so that, the boundary of the annulus $f(A)$ moves holomorphically under $h_{c}=h_{c}^{v}$. Let us extend this motion to the whole annulus $f(A)$ (using the same notation for the extension). By Lemma 2.3 this motion lifts to a holomorphic motion $H_{c}$ of $A$ over int $\mathcal{U}$ continuous up to the boundary. For any $z \in A \backslash \partial V$ and $c \in \mathcal{U}, h_{c}(f(z)) \notin \partial f(V)$, hence $H_{c}(z) \notin \partial V$. Thus, the motion of $A$ fits the tubing of $\partial V$ over $\mathcal{U}$.

Let us now consider a unicritical family $f_{c}^{q-v}: Q(c) \rightarrow V(c)$ over $\mathcal{Q}$ and a unicritical family $f_{c}^{q-v}: K(c) \rightarrow U(c)$ over $\mathcal{K}$. By Corollary 3.7 the critical value $c \mapsto f_{c}^{q-v}(0)$ is a diagonal to the corresponding tubings: of $\partial V$ over $\mathcal{Q}$ and of $\partial U$ over $\mathcal{K}$. Hence the corresponding phase-parameter map $\chi: V \rightarrow \mathcal{Q}$ maps the annulus $A$ onto the annulus $\mathcal{Q} \backslash \mathcal{K}$. By Lemma 2.1, the dilatation of this map is bounded by the dilatation of the motion $H_{c}$ over $\mathcal{Q}$, which is equal to the dilatation of $h_{c} \mid f(A)$ over $\mathcal{Q}$. By the $\lambda$-lemma, the latter is bounded by $\rho(\bmod (\mathcal{V} \backslash \mathcal{Q}))$, which implies the desired estimate.

\section{The favorite nest and the principal nest}

\subsection{The favorite nest}

Let $Q^{0}=Y^{\mathbf{n q}}$. Let $Q^{i+1}$ be the favorite child of $Q^{i}$, and let $P^{i}$ be the first child of $Q^{i}$. Let $q_{n}$ and $p_{n}$ be the depths of these puzzle pieces, i.e., $Q^{n}=Y^{q_{n}}, P^{n}=Y^{p_{n}}$. Let $k_{n}=q_{n}+p_{n-1}-q_{n-1}$ and $K^{n}=Y^{k_{n}}$. Note that

$$
f^{q_{n}-q_{n-1}}:\left(Q^{n}, K^{n}\right) \rightarrow\left(Q^{n-1}, P^{n-1}\right) .
$$

By Proposition 2.4 of [AKLS], we have

$$
\bmod \left(Q^{n} \backslash P^{n}\right)>\delta(\mathcal{O}), \quad n \geq 0 .
$$


This implies in particular that

$$
\bmod \left(Q^{n} \backslash K^{n}\right)=\frac{1}{d} \bmod \left(Q^{n-1} \backslash P^{n-1}\right)>\frac{1}{d} \delta(\mathcal{O}), \quad n \geq 1 .
$$

Let us also consider the corresponding parapuzzle pieces: $\mathcal{Q}^{n}=\mathcal{Y}^{q_{n}}, \mathcal{P}^{n}=\mathcal{Y}^{p_{n}}$, and $\mathcal{K}^{n}=\mathcal{Y}^{k_{n}}$.

Theorem 4.1. There exists $\delta>0$, depending only on $\mathcal{O}$, such that for $n \geq 2, \bmod \left(K^{n} \backslash P^{n}\right)$ $>\delta, \bmod \left(\mathcal{K}^{n} \backslash \mathcal{P}^{n}\right)>\delta$, and for $n \geq 3, \bmod \left(\mathcal{Q}^{n} \backslash \mathcal{K}^{n}\right)>\delta$.

Proof. We start with the first and second estimates. The map $f^{q_{n}-q_{n-1}}$ is unicritical on $Q^{n}$; all the more, it is unicritical on $P^{n}$. It follows that $p_{n}-q_{n} \geq q_{n}-q_{n-1}$, and hence the puzzle piece

$$
D=f^{q_{n}-q_{n-1}}\left(P^{n}\right) \subset P^{n-1}
$$

is a component of the first landing map to $Q^{n}$. It follows that $f^{p_{n-1}-q_{n-1}}(D)$ is contained in a component of the first return map to $Q^{n-1}$. In particular

$$
\begin{aligned}
\bmod \left(K^{n} \backslash P^{n}\right) & =\frac{1}{d} \bmod \left(P^{n-1} \backslash D\right) \geq \frac{1}{d^{2}} \mathrm{~m}\left(Q^{n-1}\right) \\
& \geq \frac{1}{d^{3}} \bmod \left(Q^{n-2} \backslash P^{n-2}\right) \geq \frac{1}{d^{3}} \delta(\mathcal{O}),
\end{aligned}
$$

where the last estimate follows from (4.1), while the previous one follows from Lemma 2.2 of [AKLS]. This proves the first estimate of the lemma.

For the second estimate, let us define $s<t<u<w$ as follows: $s=p_{n-1}, t=$ $q_{n}, u=k_{n}, w=p_{n}$. Then (4.3) implies that condition (1) of Lemma 3.8 is satisfied. Condition (2) of Lemma 3.8 is satisfied by Theorem 4.4 (and Remark 4.1) of [AKLS]. Applying Lemma 3.8, we get the conclusion.

For the third estimate, let $s<t<u<w$ be as follows: $s=q_{n-1}, t=k_{n-1}, u=q_{n}$, $w=k_{n}$. Then $f^{u-s}\left(Y^{w}\right)=f^{q_{n}-q_{n-1}}\left(K^{n}\right)=P^{n-1}$ is contained in $K^{n-1}$, which is a (trivial) component of the first landing map to $K^{n-1}$. By 4 4.2), condition (1) of Lemma 3.8 is satisfied.

Furthermore, $\bmod \left(\mathcal{K}^{n-1} \backslash \mathcal{Q}^{n}\right) \geq \bmod \left(\mathcal{K}^{n-1} \backslash \mathcal{P}^{n-1}\right) \geq \delta$ by the previous estimate. By Remark 3.1 and the $\lambda$-lemma, this implies that condition (2) of Lemma 3.8 is satisfied with $K=K(\mathcal{O})$. The conclusion now follows from Lemma 3.8 .

\subsection{The principal nest}

Let $V^{0}=Y^{\mathbf{n q}}$. The principal nest starting at $V^{0}$ is the nest $V^{0} \supset V^{1} \supset \cdots$ obtained by taking $V^{i+1}$ as the first child of $V^{i}$. Let $v_{i}$ be the depth of $V^{i}$, i.e., $V^{i}=Y^{v_{i}}$. Let $\mathcal{V}^{i}=\mathcal{Y}^{v_{i}}$ stand for the corresponding parapuzzle pieces. By Corollary 3.7, $\partial V^{i}$ provides us with a tubing over $\mathcal{V}^{i+1}$ with diagonal $c \mapsto f_{c}^{v_{i+1}-v_{i}}(0)$.

Let $g_{i}=g_{i, c}=R_{V^{i}}$. If the critical point returns to $V^{i+1}$, we let $s_{i}=s_{i}(c)$ be the smallest $k \geq 0$ such that $g_{i}^{k+1}(0) \in V^{i+1}$. (In other words, $s_{i}+1$ is the first return time of 
the critical point back to $V^{i+1}$ under the iterates of $g_{i}$.) If $s_{i}=0$ (that is, $g_{i}(0) \in V^{i+1}$ ), we say that the return to $V^{i}$ is central.

The map $g_{i+1}=f^{v_{i+2}-v_{i+1}}: V^{i+2} \rightarrow V^{i+1}$ admits a unicritical "Koebe extension"

$\mathbf{g}_{i+1}=\mathbf{g}_{i+1, c}: \mathbf{V}^{i+2} \rightarrow V^{i}, \quad$ where $\quad \mathbf{V}^{i+2}=Y^{v_{i+2}-v_{i+1}+v_{i}} \equiv Y^{\mathbf{v}_{i+2}} \subset V^{i+1}$.

(Note that $\mathbf{V}^{i+2}$ is a good child of $V^{i}$.) By Corollary 3.7, $\partial V^{i}$ provides us with a tubing over the parapuzzle $\mathcal{V}^{i+2} \equiv Y^{\mathbf{v}_{i+2}}$ with diagonal $c \mapsto f_{c}^{v_{i+2}-v_{i+1}}(0)$.

Lemma 4.2. We have the estimates

$$
\bmod \left(V^{i+1} \backslash V^{i+2}\right) \geq \frac{1}{d}\left(\bmod \left(V^{i} \backslash V^{i+1}\right)+s_{i} \mathrm{~m}\left(V^{i}\right)\right),
$$

$\bmod \left(\mathcal{V}^{i+1} \backslash \mathcal{V}^{i+2}\right) \geq \bmod \left(\mathcal{V}^{i+2} \backslash \mathcal{V}^{i+2}\right)>\rho\left(\bmod \left(\mathcal{V}^{i} \backslash \mathcal{V}^{i+1}\right)\right) \bmod \left(V^{i} \backslash V^{i+1}\right)$.

Proof. Let $D^{j}=Y^{d_{j}}, 1 \leq j \leq s_{i}+1$, be the pullback of $V^{i}$ under $g_{i}^{j}$, so that

$$
V^{i+1}=D^{1} \supset \cdots \supset D^{s_{i}+1}=\mathbf{V}^{i+2} .
$$

Notice that the $D^{j}$ are all children of $V^{i}$.

For $1 \leq j \leq s_{i}, f^{d_{j}-v_{i}}\left(D^{j+1}\right)$ is a non-central component of Dom $g_{i}$. It follows that $\bmod \left(V^{i} \backslash f^{d_{j}-v_{i}}\left(D^{j+1}\right)\right) \geq \mathrm{m}\left(V^{i}\right)$. Since

$$
f^{d_{j}-v_{i}}: D^{j} \backslash D^{j+1} \rightarrow V^{i} \backslash f^{d_{j}-v_{i}}\left(D^{j+1}\right)
$$

is a covering of degree $d$, we have

$$
\bmod \left(D^{j} \backslash D^{j+1}\right) \geq \frac{1}{d} \mathrm{~m}\left(V^{i}\right), \quad 1 \leq j \leq s_{i} .
$$

Moreover, $f^{v_{i+2}-v_{i+1}}: \mathbf{V}^{i+2} \backslash V^{i+2} \rightarrow V^{i} \backslash V^{i+1}$ is a covering of degree $d$ as well. Hence

$$
\bmod \left(\mathbf{V}^{i+2} \backslash V^{i+2}\right)=\frac{1}{d} \bmod \left(V^{i} \backslash V^{i+1}\right) .
$$

Putting (4.7), 4.8) together with the Grötzsch inequality, we get (4.4).

Applying Lemma 3.9 to the nest $V^{i+2} \subset \mathbf{V}^{i+2} \subset V^{i+1} \subset V^{i}$, we obtain

$$
\bmod \left(\mathcal{V}^{i+2} \backslash \mathcal{V}^{i+2}\right)>\rho\left(\bmod \left(\mathcal{V}^{i} \backslash \mathcal{V}^{i+2}\right)\right) \bmod \left(V^{i} \backslash V^{i+1}\right) .
$$

Since $\mathcal{V}^{i+1} \supset \mathcal{V}^{i+2}, 4.5$ follows.

Define $0=i_{0}<i_{1}<\cdots$ to be the sequence of levels such that for $j>0$ the return to $i_{j}-1$ is non-central, i.e., $g_{i_{j}-1}(0) \notin V^{i_{j}}$.

Lemma 4.3. Let $i_{j-1} \leq s<i_{j}$. Then we have the estimates

$$
\begin{aligned}
& \bmod \left(V^{i_{j}} \backslash V^{i_{j}+1}\right) \geq \frac{1}{d} \bmod \left(V^{s} \backslash V^{s+1}\right), \\
& \bmod \left(\mathcal{V}^{i_{j}} \backslash \mathcal{V}^{i_{j}+1}\right)>\rho\left(\bmod \left(\mathcal{V}^{s} \backslash \mathcal{V}^{s+1}\right)\right) \bmod \left(V^{s} \backslash V^{s+1}\right) .
\end{aligned}
$$


Proof. Let $n$ be the first moment such that $f^{n}(0) \in V^{s} \backslash V^{s+1}$, and let $m>0$ be the first moment such that $f^{n+m}(0) \in V^{s+1}$. Then $Q=Y^{v_{s}+n+m}$ is the favorite child of $V^{s}$.

We have $Q \supset V^{i_{j}+1}$ since $V^{s} \supset V^{i_{j}}$ and $n+m$ is not great than the first return time to $V^{i_{j}}$. A similar argument gives $f^{n+m}\left(V^{i_{j}+1}\right) \subset V^{s+1}$. Hence

$$
\bmod \left(Q \backslash V^{i_{j}+1}\right) \geq \frac{1}{d} \bmod \left(V^{s} \backslash V^{s+1}\right) .
$$

Since $Q \subset Y^{v_{s}+n}=V^{i_{j}}$, the first statement follows.

The second statement follows from Lemma 3.9 applied to the nest

$$
K \subset Q \subset V^{s+1} \subset V^{s},
$$

where $K$ is the friend of $Q$ (since $f^{n+m}\left(V^{i_{j}+1}\right) \subset V^{s+1}$ implies $\left.V^{i_{j}+1} \subset K\right)$.

Lemma 4.4. If $V^{i_{j}-1} \subset Q^{n}$ and $V^{i_{j}+1}$ is defined then $V^{i_{j}+1} \subset Q^{n+1}$.

Proof. Recall that $q_{n+1}=q_{n}+m$ where $m$ is minimal with $f^{m}(0) \in P^{n}$ and $\left\{f^{k}(0)\right.$ : $1 \leq k \leq m\} \cap Q^{n} \backslash P^{n} \neq \emptyset$. Clearly $V^{i_{j}} \subset P^{n}$, so we just have to show that $\left\{f^{k}(0): 1 \leq\right.$ $\left.k \leq v_{i_{j}+1}-v_{i_{j}}\right\} \cap Q^{n} \backslash P^{n} \neq \emptyset$, as this implies $q_{n+1}=q_{n}+m \leq v_{i_{j}}+m \leq v_{i_{j}}+v_{i_{j}+1}-v_{i_{j}}$.

Let $k \geq 1$ be minimal such that $f^{k}(0) \in Q^{n} \backslash P^{n}$. Then $k=l_{0}\left(p_{n}-q_{n}\right)$ for some $l_{0}>0$, and $R_{Q^{n}}^{l}(0) \in Y^{q_{n}+\left(l_{0}-l\right)\left(p_{n}-q_{n}\right)} \backslash Y^{p_{n}+\left(l_{0}-l\right)\left(p_{n}-q_{n}\right)}, 1 \leq l \leq l_{0}$.

Since $R_{V^{i_{j}-1}}(0) \notin V^{i_{j}}$, we also have $R_{Q^{n}}(0) \notin V^{i_{j}}$, so that $V^{i_{j}} \subset Y^{q_{n}+\left(l_{0}-1\right)\left(p_{n}-q_{n}\right)}$. This clearly implies that $R_{Q^{n}}^{l}(0) \notin V^{i_{j}}, 1 \leq l \leq l_{0}$, so $v_{i_{j}+1}-v_{i_{j}} \geq k$, as desired.

Theorem 4.5. There exists $\delta>0$, depending only on $\mathcal{O}$, such that

(1) $\bmod \left(V^{i_{j}} \backslash V^{i_{j}+1}\right)>\delta$,

(2) $\bmod \left(\mathcal{V}^{i_{j}} \backslash \mathcal{V}^{i_{j}+1}\right)>\delta$.

Proof. By Lemma 3.3 the first statement holds for $j=0$. Since $\mathcal{V}^{0}=\mathcal{Y}^{\mathbf{n q}}=\Omega^{\mathbf{n}}$ and $\mathcal{V}^{1} \subset \mathcal{Y}^{\mathbf{n q}+1}=\mathcal{O}$, we have $\bmod \left(\mathcal{V}^{0} \backslash \mathcal{V}^{1}\right) \geq \bmod \left(\Omega^{\mathbf{n}} \backslash \mathcal{O}\right)=\delta(\mathcal{O})$, so the second statement also holds for $j=0$.

By Lemmas 4.4 and 4.3 , it is enough to show that for every $n \geq 3$ such that $Q^{n+1}$ is defined, if $k \geq 0$ is maximal such that $V^{k} \supset Q^{n}$ then

$$
\bmod \left(V^{k+1} \backslash V^{k+2}\right), \bmod \left(\mathcal{V}^{k+1} \backslash \mathcal{V}^{k+2}\right)>\delta .
$$

Note first that since $P^{n}$ and $V^{k+2}$ are the first children of $Q^{n}$ and $V^{k+1}$ respectively, we have $V^{k+2} \subset P^{n}$. Recall the definition of $K^{n}$ given at the beginning of Section 4.1 . $K^{n}=Y^{q_{n}+p_{n-1}-q_{n-1}}$. If $V^{k+1} \supset K^{n}$, then

$$
\bmod \left(V^{k+1} \backslash V^{k+2}\right) \geq \bmod \left(K^{n} \backslash P^{n}\right) \quad \text { and } \bmod \left(\mathcal{V}^{k+1} \backslash \mathcal{V}^{k+2}\right) \geq \bmod \left(\mathcal{K}^{n} \backslash \mathcal{P}^{n}\right) \text {. }
$$

If $K^{n} \supset V^{k+1}$, then $\bmod \left(V^{k} \backslash V^{k+1}\right) \geq \bmod \left(Q^{n} \backslash K^{n}\right)$ and $\bmod \left(\mathcal{V}^{k} \backslash \mathcal{V}^{k+1}\right) \geq$ $\bmod \left(\mathcal{Q}^{n} \backslash \mathcal{K}^{n}\right)$, so by Lemma 4.2 ,

$$
\begin{aligned}
& \bmod \left(V^{k+1} \backslash V^{k+2}\right) \geq \frac{1}{d} \bmod \left(Q^{n} \backslash K^{n}\right), \\
& \bmod \left(\mathcal{V}^{k+1} \backslash \mathcal{V}^{k+2}\right)>\rho\left(\bmod \left(\mathcal{Q}^{n} \backslash \mathcal{K}^{n}\right)\right) \bmod \left(Q^{n} \backslash K^{n}\right) .
\end{aligned}
$$

In either case, the result follows from Theorem 4.1 
Remark 4.1. In [L3], [L4], it is shown that if $d=2$ then one has better estimates

(1) $\bmod \left(V^{i_{j}} \backslash V^{i_{j}+1}\right)>(j+1) \delta$,

(2) $\bmod \left(\mathcal{V}^{i_{j}} \backslash \mathcal{V}^{i_{j}+1}\right)>(j+1) \delta$.

Remark 4.2 (Beau bounds). It follows from the arguments in [KL1], [KL2], [AKLS], and this work that there exists $\delta>0$ (depending on the degree, but not on $\mathcal{O}$ ) such that for every $j$ sufficiently large (depending on $\mathcal{O}$ ) one has

(1) $\bmod \left(V^{i_{j}} \backslash V^{i_{j}+1}\right)>\delta$,

(2) $\bmod \left(\mathcal{V}^{i_{j}} \backslash \mathcal{V}^{i_{j}+1}\right)>\delta$.

\section{Slow recurrence}

If $c \in \mathcal{M}$ is not combinatorially recurrent then either $c$ has a non-repelling fixed point, or $f_{c}$ is satellite renormalizable, or $f_{c}$ is semi-hyperbolic (that is, its critical point is nonrecurrent and belongs to the Julia set). It is well known that the set of semi-hyperbolic parameters has zero Lebesgue measure. Indeed, in $[\mathrm{RL}]$ a more precise version of the following is proved:

Theorem 5.1. If $c \in \mathcal{M}$ is a semi-hyperbolic parameter then $c$ is a Lebesgue density point of the complement of $\mathcal{M}$.

In particular, almost every parameter in some $\mathcal{V}^{n+1}$ is either in the complement of $\mathcal{M}$ or is combinatorially recurrent. For real parameters, the corresponding statement has been proved in [Sa]: the set of semi-hyperbolic parameters $c \in \mathcal{M} \cap \mathbb{R}$ has zero onedimensional Lebesgue measure. We will now concentrate on the analysis of combinatorially recurrent parameters.

Remark 5.1. A proof that the set of semi-hyperbolic parameters has zero Lebesgue measure can also be obtained along the lines of the approach we follow for combinatorially recurrent parameters given below.

\subsection{Positive probability of slow recurrence}

Lemma 5.2. For any $\delta>0$ and $\lambda>0$ there exist $\epsilon=\epsilon(d, \delta)$ and $K=K(d, \delta, \lambda)$ with the following property. Assume that for some parapuzzle piece $\mathcal{V}^{n}$,

$$
\bmod \left(\mathcal{V}^{n} \backslash \mathcal{V}^{n+1}\right)>\delta \quad \text { and } \bmod \left(V^{n}(c) \backslash V^{n+1}(c)\right)>\delta, \quad c \in \mathcal{V}^{n+1} .
$$

Then for every $c \in \mathcal{V}^{n+k+3}$ such that $s_{n+i} \geq(1+i) K, i=0,1, \ldots, k$, we have:

(1) $\bmod \left(V^{n+i} \backslash V^{n+i+1}\right)>\max \{\epsilon,(i-1) \lambda\}$,

(2) $\bmod \left(\mathcal{V}^{n+i} \backslash \mathcal{V}^{n+i+1}\right)>\max \{\epsilon,(i-2) \lambda\}$,

(3) $\bmod \left(\mathcal{V}^{n+i+2} \backslash \mathcal{V}^{n+i+2}\right)>\max \{\epsilon,(i-1) \lambda\}$,

for $i=0,1, \ldots, k+1$. 
Proof. The inequalities $\bmod \left(V^{n+i+1} \backslash V^{n+i+2}\right) \geq \frac{1}{d}\left(1+s_{n+i}\right) \mathrm{m}\left(V^{n+i}\right)$, given by 4.4$)$, and $\mathrm{m}\left(V^{n+i+1}\right) \geq \frac{1}{d} \bmod \left(V^{n+i} \backslash V^{n+i+1}\right)$, given by Lemma 2.2 of [AKLS], imply the estimate $\bmod \left(V^{n+i} \backslash V^{n+i+1}\right)>\delta_{i}(1+i)$, where $\delta_{0}=\delta, \delta_{1}=\delta / d$, and $\inf _{i \geq 1} \delta_{i}$ goes to infinity with $K$ (given $\delta$ and $d$ fixed). The first estimate follows.

Together with (4.5), it implies the rest.

Given a sequence of disjoint sets $X^{n}, n \geq 0$, on a probability space $\mathcal{V}$, we let $\mathbb{X}^{n}=$ $\bigcup_{k<n} X^{n}, \mathbb{X} \equiv \bigcup_{k \geq 0} X^{k}$. Below we will make use of the following general formula:

$$
1-\mathbb{P}(\mathbb{X})=\prod_{n \geq 0}\left(1-\mathbb{P}\left(X^{n} \mid \mathcal{V} \backslash \mathbb{X}^{n}\right)\right)
$$

(where $\mathbb{P}$ stands for probability or conditional probability). Indeed, letting $A^{n}=\mathcal{V} \backslash \mathbb{X}^{n}$, $A \equiv \bigcap_{n \geq 0} A^{n}=\mathcal{V} \backslash X$, we have $A^{0} \supset A^{1} \supset \cdots \supset A$, and

$$
\mathbb{P}(A)=\prod_{n \geq 0} \mathbb{P}\left(A^{n+1} \mid A^{n}\right)=\prod_{n \geq 0} \mathbb{P}\left(\mathcal{V} \backslash X^{n} \mid \mathcal{V} \backslash \mathbb{X}^{n}\right),
$$

which yields 5.1$)$

Lemma 5.3. Assume that for some parameter $c_{0}$,

$$
\bmod \left(\mathcal{V}^{n} \backslash \mathcal{V}^{n+1}\right)>\delta, \quad \bmod \left(V^{n} \backslash V^{n+1}\right)>\delta .
$$

Let $Z_{\mathrm{nr}} \subset \mathcal{V}^{n+1}$ be the set of parameters which are not combinatorially recurrent. Fix some $K>0$ as in Lemma 5.2 and let $Z_{\mathrm{sr}} \subset \mathcal{V}^{n+1}$ be the set of combinatorially recurrent parameters for which $s_{n+k} \geq(1+k) K, k \geq 0.6$ Then $\mathbb{P}\left(Z_{\mathrm{sr}} \cup Z_{\mathrm{nr}} \mid \mathcal{V}^{n+1}\right)>$ $\epsilon(\delta, d, K)>0$.

Proof. We can assume that $K$ is larger than the $K(\delta, d)$ given by Lemma 5.2 Let $t_{k}=$ $(1+k) K$, and let $\mathbb{X}=\mathcal{V}^{n+1} \backslash\left(Z_{\mathrm{sr}} \cup Z_{\mathrm{nr}}\right)$. For $k \geq 0,0 \leq j<t_{k}$, let $X^{k, j} \subset \mathbb{X}$ be the set of all $c \in \mathbb{X}$ such that $s_{n+i} \geq t_{i}, 0 \leq i<k$ and $s_{n+k}=j$. Notice that $\mathbb{X}=\bigsqcup_{(k, j)} X^{k, j}$. We order the pairs $(k, j)$ lexicographically. As above, let

$$
\mathbb{X}^{k, j}=\bigcup_{\left(k^{\prime}, j^{\prime}\right)<(k, j)} X^{k^{\prime}, j^{\prime}}
$$

Notice that for $c \in X^{k, j}$, we have

$$
\mathcal{V}^{n+k+2}(c) \cap \mathbb{X}^{k, j}=\emptyset
$$

while

$$
\mathcal{V}^{n+k+2}(c) \cap X^{k, j} \subset \mathcal{V}^{n+k+2}(c) .
$$

Indeed, for $\tilde{c} \in \mathcal{V}^{n+k+2}(c)$, we have $s_{n+i}(\tilde{c})=s_{n+i}(c) \geq t_{n+i}$ for $i<k$, while $s_{n+k}(\tilde{c}) \geq$ $s_{n+k}(c)=j$, with equality attained iff $\tilde{c} \in \mathcal{V}^{n+k+2}(c)$.

6 Label "sr" stands for "slow recurrent". 
Together with 5.1, 5.2) gives us

$$
\begin{aligned}
\mathbb{P}\left(Z_{\mathrm{nr}} \cup Z_{\mathrm{sr}} \mid \mathcal{V}^{n+1}\right) & =1-\mathbb{P}\left(\mathbb{X} \mid \mathcal{V}^{n+1}\right)=\prod_{(k, j)}\left(1-\mathbb{P}\left(X^{k, j} \mid \mathcal{V}^{n+1} \backslash \mathbb{X}^{k, j}\right)\right) \\
& \geq \prod_{(k, j)}\left(1-\sup _{c \in X^{k, j}} \mathbb{P}\left(X^{k, j} \mid \mathcal{V}^{n+k+2}(c)\right)\right.
\end{aligned}
$$

It is thus enough to prove an estimate such as

$$
\mathbb{P}\left(X^{k, j} \mid \mathcal{V}^{n+k+2}(c)\right) \leq e^{-(1+k) \epsilon}, \quad c \in X^{k, j},
$$

for some $\epsilon=\epsilon(\delta, d)$. But this follows from (5.3) and the estimate

$$
\bmod \left(\mathcal{V}^{n+k+2}(c) \backslash \mathcal{V}^{n+k+2}(c)\right) \geq(1+k) \epsilon
$$

of the previous lemma.

Remark 5.2. The above proof can be easily refined as follows. One can define $Z_{\mathrm{sr}}$ as the set of combinatorially recurrent parameters $c \in \mathcal{V}^{n+1}$ for which the sequence $s_{n+i}$ satisfies $s_{n}, s_{n+1}, s_{n+2} \geq K$ and $s_{n+i+1} \geq 2^{s_{n_{i}}}$ for $i \geq 2$ (thus displaying "torrential growth" in the terminology of [AM1] $)$. We would still obtain $\mathbb{P}\left(Z_{\mathrm{nr}} \cup Z_{\mathrm{sr}} \mid \mathcal{V}^{n+1}\right)>\epsilon$.

Let $\mathcal{S} \subset \mathcal{M}$ be the set of combinatorially recurrent parameters $c$ such that $s_{n}$, $\bmod \left(\mathcal{V}^{n}(c) \backslash \mathcal{V}^{n+1}(c)\right)$ and $\bmod \left(V^{n}(c) \backslash V^{n+1}(c)\right)$ grow at least linearly with $n$.

Let $Z_{\mathrm{r}}$ be the set of combinatorially recurrent non-renormalizable parameters in $\mathcal{M}$.

Corollary 5.4. For $c \in Z_{\mathrm{r}}$, there exist parapuzzle pieces $\mathcal{V}^{n+1}(c)$ of arbitrarily small diameter such that $\mathbb{P}\left(\mathcal{M} \backslash \mathcal{S} \mid \mathcal{V}^{n+1}(c)\right)<1-\delta$, with $\delta=\delta(\mathcal{O}) .7$

Proof. For $c \in Z_{\mathrm{r}}$, the sequence $i_{j}$ in Theorem 4.5 is infinite. By the Rigidity Theorem of [AKLS] (or directly from Theorem 4.5), the parapuzzle pieces $\mathcal{V}^{i_{j}}(c)$ shrink to $c$. We can now apply Lemma 5.3 with $n=i_{j}$, which implies the statement (since by Theorem 5.1 combinatorially non-recurrent parameters in $\mathcal{V}^{n+1}$ are almost surely outside $\mathcal{M}$ ).

In order to exploit the previous corollary, we will need the following "Density Points Argument". Let us consider a measurable set $X \subset \mathbb{C}$ such that for almost every $x \in X$ there exists a sequence $X^{n}(x) \subset \mathbb{C}$ of measurable sets containing $x$ such that diam $X^{n}(x) \rightarrow 0$. Assume that any two $X^{n}(x), X^{m}(y)$ are either nested or disjoint. Then $\lim \mathbb{P}\left(X \mid X^{n}(x)\right)$ $=1$ for almost every $x \in X$. This is a particular case of the standard generalization of the Lebesgue Density Points Theorem (which assumes that the family $\left\{X^{n}(x)\right\}_{x, n}$ satisfies the Besicovitch Covering Property), and can also be seen as a direct consequence of the Martingale Convergence Theorem.

Corollary 5.5. For almost every $c \in \mathcal{M}$, either $f_{c}$ has an attracting fixed point, or $f_{c}$ is renormalizable, or $c \in \mathcal{S}$.

Proof. It is enough to show that $\mathcal{S}$ has full Lebesgue measure in $Z_{\mathrm{r}}$. For fixed $n$, the parapuzzle pieces $\mathcal{V}^{n}(\tilde{c})$ define a partition of $Z_{\mathrm{r}}$. Since the $\mathcal{V}^{n}(c)$ shrink to $c$ for any $c \in Z_{\mathrm{r}}$, we can apply the Density Points Argument, which implies that $\lim \mathbb{P}\left(Z_{\mathrm{r}} \backslash \mathcal{S} \mid \mathcal{V}^{n}(c)\right)=1$ for almost every $c \in Z_{\mathrm{r}} \backslash \mathcal{S}$. But by Corollary 5.4, this cannot happen for $c \in Z_{\mathrm{r}}$.

7 Actually $\delta>0$ does not depend on $c$, not even via the Misiurewicz wake: see Remark 4.2 


\subsection{Real parameters}

Our entire discussion goes through for real parameters as well, without changes. However there are no parameters in $\mathcal{V}^{n+1} \cap \mathbb{R} \backslash \mathcal{M}$, so that we can state the following stronger version of Corollary 5.4 .

Corollary 5.6. There exists $\delta>0$ such that for $c \in Z_{\mathrm{r}} \cap \mathbb{R}$, there exist parapuzzle pieces $\mathcal{V}^{n+1}(c)$ of arbitrarily small diameter such that $\mathbb{P}\left(\mathcal{S} \mid \mathcal{V}^{n+1}(c) \cap \mathbb{R}\right)>\delta$.

Corollary 5.7. For almost every $c \in \mathcal{M} \cap \mathbb{R}$, either $f_{c}$ has an attracting fixed point, or $f_{c}$ is renormalizable, or $c \in \mathcal{S}$.

Parameters in $\mathcal{S} \cap \mathbb{R}$ have exponential decay of geometry, that is, the ratios $\lambda_{n}$ between the lengths of $V^{n+1} \cap \mathbb{R}$ and $V^{n} \cap \mathbb{R}$ satisfy $\lambda_{n}<C e^{-\epsilon n}$ for some $C, \epsilon>0$. Hence

$$
\sum \lambda_{n}^{1 / d}<\infty
$$

and by the Martens-Nowicki Criterion [MN] the maps $P_{c}, c \in \mathcal{S}$, are stochastic (that is, they have an absolutely continuous invariant measure).

Remark 5.3. In [BSS1] it is shown that the decay of geometry (that is, $\lambda_{n} \rightarrow 0$ ) already implies the existence of an absolutely continuous invariant measure.

Corollary 5.8. The set of non-renormalizable stochastic parameters $c \in \mathcal{M} \cap \mathbb{R}$ has positive Lebesgue measure.

Corollary 5.9. Almost every non-renormalizable parameter $c \in \mathcal{M} \cap \mathbb{R}$ is either regular or stochastic.

Remark 5.4. Corollary 5.8, in the case $d=2$, was obtained in [J]. The generalization to the higher degree case is well known (see [T, Theorem 2], which follows the approach of $[\overline{B C}])$. Our proof is rather different.

Corollary [5.9, in the case $d=2$, was obtained in [L4] and is new in the higher degree case.

\section{Conclusion}

By now, we have carried out all the extra work needed for the higher degree case: once we know that the phase-parameter geometry almost surely decays (Corollaries 5.5 and 5.77, the further argument is the same as in the quadratic case. For the reader's convenience, below we will briefly elaborate this statement. 


\subsection{Real parameters}

6.1.1. Collet-Eckmann property (Theorem 1.1). Standard renormalization considerations reduce the analysis of exactly $n$-times renormalizable parameters with some fixed combinatorics to the analysis of non-renormalizable parameters in a "Multibrot-like family". The analysis of Multibrot-like families is parallel to the one we have done (see [L4] which deals directly, in the case $d=2$, with Mandelbrot-like families), and one reaches the same theorems, with the difference that all constants may depend on the geometry of the Multibrot-like family under consideration.

Since a renormalizable map is Collet-Eckmann if and only if its renormalization is, Theorem 1.1 follows from the statement that (in a Multibrot-like family) real nonrenormalizable parameters are almost surely either regular or Collet-Eckmann. In view of Corollary 5.7, this is reduced to the following result:

Theorem 6.1. The Collet-Eckmann parameters have full (one-dimensional) Lebesgue measure in $\mathcal{S} \cap \mathbb{R}$.

This result follows from the statistical argument of [AM1]: as pointed out in Remark 2.1 of that paper, the statistical argument applies for any $d$ to the set of parameters satisfying the following properties:

- $\liminf s_{n} \geq 1$;

- exponential decay of the real phase geometry (meaning that the ratios of the lengths of the real traces of $V^{n+1}$ and $V^{n}$ decay exponentially);

- growth of the parameter moduli $\bmod \left(\mathcal{V}^{n} \backslash \mathcal{V}^{n+1}\right)$.

All these conditions hold for non-renormalizable parameters $c \in \mathcal{S}$ (the exponential decay of geometry follows from the linear growth of the phase $\left.\operatorname{moduli} \bmod \left(V^{n} \backslash V^{n+1}\right)\right)$.

6.1.2. Further statistical properties. The statistical analysis of [AM1] and [AM3] goes far beyond the Collet-Eckmann property, and gives a very detailed description of maps in $\mathcal{S} \cap \mathbb{R}$. As for the Collet-Eckmann property, it can be directly applied to the higher degree case:

Theorem 6.2. For almost every $c \in \mathbb{R}$ such that $f_{c}$ is not regular or infinitely renormalizable:

(1) The critical point is polynomially recurrent with exponent 1 :

$$
\limsup \frac{-\ln \left|f_{c}^{n}(0)\right|}{\ln n}=1 \text {. }
$$

(2) The critical orbit is equidistributed with respect to the absolutely continuous invariant measure $\mu$ :

$$
\lim \frac{1}{n} \sum_{i=0}^{n-1} \phi\left(f_{\lambda}^{i}(0)\right)=\int \phi d \mu
$$

for any continuous function $\phi: I \rightarrow \mathbb{R}$. 
(3) The Lyapunov exponent of the critical value, $\lim \frac{1}{n} \ln \left|D f^{n}(f(0))\right|$, exists and coincides with the Lyapunov exponent of $\mu$.

(4) The Lyapunov exponent of any periodic point $p$ contained in supp $\mu$ is determined (via an explicit formula) by combinatorics (more precisely, by the itineraries of $p$ and of the critical point).

\subsection{Zero area (Theorem 1.3}

Again, by renormalization considerations, Theorem 1.3 reduces to the statement that (in a Multibrot-like family) almost every non-renormalizable parameter is regular. In view of Corollary 5.5. it is thus enough to prove the following statement:

Theorem 6.3. The set $\mathcal{S}$ has zero area.

Proof. Fix an arbitrary $c_{0} \in \mathcal{S}$ and let $\mathcal{V}^{n+1}=\mathcal{V}^{n+1}\left(c_{0}\right)$. For $c \in \mathcal{V}^{n+1}$, let $g_{n, c}$ denote the first return map to $V^{n}$ under iteration by $f_{c}$, and let $V_{*}^{n}(c)$ be the component of $D_{n}(c)=\operatorname{Dom}\left(g_{n, c}\right)$ containing the critical value $\left.g_{n, \tilde{c}}(0)\right]^{8} \operatorname{Set}$

$$
\mathcal{V}_{*}^{n+1}=\left\{c \in \mathcal{V}^{n+1}: g_{n, c}(0) \in V_{*}^{n}(c)\right\}
$$

By the "Density Points Argument" of the previous section, it is sufficient to show that

$$
\lim \sup \mathbb{P}\left(\mathcal{S} \mid \mathcal{V}_{*}^{n+1}\right)<1 .
$$

For $c \in \mathcal{V}^{n+1}$, let $Z^{n+1}(c)$ be the union of the boundaries of the puzzle pieces that are subordinate to depth $v_{n+1}$ (and hence move holomorphically over $\mathcal{V}^{n+1}$ ). Persistent puzzle pieces include all components of $D_{n}(c)$. By Słodkowski's Theorem, the holomorphic motion of $Z^{n+1}$ extends to a holomorphic motion $h$ of the whole complex plane $\mathbb{C}$.

The map $\psi: c \mapsto g_{n, c}(0)$ is a diagonal to the tubing of $\partial V_{*}^{n}$ over $\mathcal{V}_{*}^{n+1}$, so $h$ and $\psi$ give rise to a phase-parameter map $\chi_{n}: V_{*}^{n} \rightarrow \mathcal{V}_{*}^{n+1}$. Since

$$
\bmod \left(V^{n}(c) \backslash V_{*}^{n}(c)\right) \geq \delta n
$$

for all $c \in \mathcal{V}^{n+1}$, the first statement of Lemma 2.2 implies that $\bmod \left(\mathcal{V}^{n+1} \backslash \mathcal{V}_{*}^{n+1}\right) \rightarrow \infty$, so by the $\lambda$-lemma, $\chi_{n}$ is $\gamma_{n}$-qc, where $\gamma_{n} \rightarrow 19$

Given two measurable sets $X \subset Y$ and a bi-measurable injection $\phi: Y \rightarrow \mathbb{C}$, we let

$$
\mathbb{P}_{\phi}(X \mid Y)=\mathbb{P}(\phi(X) \mid \phi(Y))
$$

be the $\phi$-pullback of the conditional probability. Let $\gamma>1$. Given a Jordan disk $V$ and a measurable set $X \subset D$, let us define the $\gamma$-capacity $\mathbb{P}_{\gamma}(X \mid V)$ as follows:

$$
\mathbb{P}_{\gamma}(X \mid V)=\sup \mathbb{P}_{\phi}(X \mid V)
$$

\footnotetext{
8 In what follows we let $D_{n}=D_{n}\left(c_{0}\right)$, and use the similar convention for other objects moving over $\mathcal{V}^{n+1}$.

9 This kind of rules relating the dynamical and parameter objects are described in [AM2] as the phase-parameter relation. For most purposes, one can use these rules axiomatically.
} 
where $\phi$ ranges over all $\gamma$-quasiconformal homeomorphisms $V \rightarrow \phi(V) \Subset \mathbb{C}$. Clearly, the $\gamma$-capacity is a conformal invariant. Let $\alpha_{n}=\mathbb{P}_{\gamma}\left(D_{n} \mid V^{n}\right)$.

For $n>0$, the set $D_{n}$ is "uniformly porous" in $V^{n}$ in the following sense: There exist $K>0, \mu>0$ and $\eta>0$ such that any component $W$ of $D_{n}$ is contained in the nest of two topological disks, $W \subset W^{\prime} \subset W^{\prime \prime} \subset V^{n}$, such that:

- $\bmod \left(W^{\prime \prime} \backslash W^{\prime}\right) \geq \mu$;

- $W^{\prime}$ is a $K$-quasidisk;

- $\mathbb{P}\left(D_{n} \mid W^{\prime}\right) \leq 1-\eta$.

To obtain such a nest, take the return map $g_{n}=g_{n, c}: W \rightarrow V^{n}$, extend it to a branched covering $\hat{g}_{n}: W^{\prime \prime} \rightarrow V^{n-1}$ of degree $d$ or 1 , and let $W^{\prime}$ be the pullback by $\hat{g}_{n}$ of a big intermediate quasidisk $U, V^{n} \Subset U \Subset V^{n-1}$. Since $D_{n-1}$ is not dense in $U$ (once $U$ is sufficiently large), the Koebe Distortion Theorem implies that $D^{n}$ has a gap of a definite size in $W^{\prime}$ (compare Lemma B.3 of [AM2]).

Uniform porosity implies that $\alpha_{n}<1$ for $n>0$ (making use of the Besicovitch Covering Lemma).

Since $g_{n, c}: V_{*}^{n}(c) \rightarrow V^{n}(c)$ is a conformal map for $c \in \mathcal{V}^{n+1}$, the connected components of the set

$$
\Delta_{n}(c)=\left(g_{n, c} \mid V_{*}^{n}(c)\right)^{-1}\left(D_{n}(c)\right)
$$

are puzzle pieces which are subordinate to depth $n+1$ and hence $\Delta_{n}(c)$ is respected by the holomorphic motion $h$. Moreover, for $c \in \mathcal{S} \cap \mathcal{V}^{n+1}, g_{n, c}(0) \in \Delta_{n}(c)$. It follows that $\mathcal{S} \cap \mathcal{V}_{*}^{n+1}$ is contained in the image of $\Delta_{n}$ under the phase-parameter map $\chi_{n}: V_{*}^{n} \rightarrow$ $\mathcal{V}_{*}^{n+1}$. Since this map is $\gamma$-quasiconformal for large $n, \mathbb{P}\left(\mathcal{S} \mid \mathcal{V}_{*}^{n+1}\right) \leq \alpha_{n}$ by definition of capacity. Thus, to prove 6.1), it is enough to show that

$$
\lim \sup \alpha_{n}<1 .
$$

We will obtain this by means of the following simple statistical argument.

Let $O^{n+1}=\left(g_{n} \mid V^{n+1}\right)^{-1}\left(D_{n}\right)$. For each connected component $W$ of $O^{n+1}$, we have

$$
\bmod \left(V^{n+1} \backslash W\right) \geq \frac{1}{d^{2}} \bmod \left(V^{n-1} \backslash V^{n}\right) \geq \delta n .
$$

Call a component of $O^{n+1}$ critical if it contains 0 and precritical if its image under $g_{n}$ contains 0 . Let $E^{n+1}$ be the union of critical and precritical components. If $s_{n}=0$ (the central return case) then $E^{n+1}=V^{n+2}$; otherwise $E^{n+1}$ consists of $d+1$ puzzle pieces. In any case, $E^{n+1}$ is the union of at most $d+1$ puzzle pieces $W_{i} \subset V^{n+1}$, each satisfying 6.3. It follows that

$$
\mathbb{P}_{\gamma}\left(E^{n+1} \mid V^{n+1}\right) \equiv \epsilon_{n+1} \leq e^{-\delta n} .
$$

Furthermore, if $W$ is a connected component of $O^{n+1} \backslash E^{n+1}$ then $g_{n}^{2}: W \rightarrow V^{n}$ is a conformal map, and $g_{n}^{2}\left(W \cap D_{n+1}\right) \subset D_{n}$. It follows that if $\phi: V^{n+1} \rightarrow \phi\left(V^{n+1}\right) \Subset \mathbb{C}$ is a $\gamma$-qc homeomorphism then for any such component $W$ we have $\mathbb{P}_{\phi}\left(D_{n+1} \mid W\right) \leq \alpha_{n}$ (by the definition of capacity). Hence

$$
\mathbb{P}_{\phi}\left(D^{n+1} \mid O^{n+1} \backslash E^{n+1}\right) \leq \alpha_{n}
$$


so that

$$
\mathbb{P}_{\phi}\left(D^{n+1} \backslash E^{n+1} \mid V^{n+1}\right) \leq\left(1-\mathbb{P}_{\phi}\left(E^{n+1} \mid V^{n+1}\right)\right) \alpha_{n}
$$

Thus,

$$
\begin{aligned}
\mathbb{P}_{\phi}\left(D_{n+1} \mid V^{n+1}\right) & =\mathbb{P}_{\phi}\left(E^{n+1} \mid V^{n+1}\right)+\mathbb{P}_{\phi}\left(D_{n+1} \backslash E^{n+1} \mid V^{n+1}\right) \\
& \leq \mathbb{P}_{\phi}\left(E^{n+1} \mid V^{n+1}\right)+\left(1-\mathbb{P}_{\phi}\left(E^{n+1} \mid V^{n+1}\right)\right) \alpha_{n} \\
& =\alpha_{n}+\left(1-\alpha_{n}\right) \mathbb{P}_{\phi}\left(E^{n+1} \mid V^{n+1}\right) .
\end{aligned}
$$

Taking the supremum over all $\phi$ under consideration, we obtain

$$
\alpha_{n+1} \leq \alpha_{n}+\left(1-\alpha_{n}\right) \epsilon_{n+1},
$$

so

$$
\frac{1-\alpha_{n+1}}{1-\alpha_{n}} \geq 1-\epsilon_{n+1} \geq 1-e^{-\delta n}
$$

which yields 6.2.

\subsection{Porosity of $\mathcal{M}$ (Theorem 1.4)}

If $c$ is not combinatorially recurrent, then by Theorem 5.1, $c$ is a Lebesgue density point of the complement of $\mathcal{M}$.

So, assume that $c$ is combinatorially recurrent. By Theorem $4.5 \cap \mathcal{Y}^{n}(c)=\{c\}$, and by Corollary 5.4 and Theorem 6.3. $\lim \inf \mathbb{P}\left(\mathcal{M} \mid \mathcal{Y}^{n}(c)\right)<1$. This is not enough, though, to conclude that $c$ is not a Lebesgue density point of $\mathcal{M}$, since the $\mathcal{Y}^{n}(c)$ do not in general have a bounded shape (where a set $K \subset \mathbb{C}$ is said to have $C$-bounded shape if it contains a round disk of radius $(1 / C) \operatorname{diam}(K)) \cdot{ }^{10}$ However, the following lemma will allow us to replace them with shrinking domains of bounded shape.

Lemma 6.4. For every $\delta>0$, there exists $\kappa>1$ with the following property. Let $D$ be a Jordan disk and let $x \in D$. Then there exists $r>0$ such that $\mathbb{D}_{r}(x) \subset D$ and for every Jordan disk $\Delta \subset D$ that intersects both $\mathbb{D}_{r}(x)$ and $\partial \mathbb{D}_{\kappa r}(x)$, we have $\bmod (D \backslash \Delta)<\delta$.

Proof. Let $\psi:(D, x) \rightarrow(\mathbb{D}, 0)$ be the Riemann map, and let $r$ be the maximal radius such that $\psi\left(\mathbb{D}_{r}(x)\right) \subset \mathbb{D}_{1 / 2}$.

Notice that if $B \subset \mathbb{D}$ is a Jordan disk with $\bmod (\mathbb{D} \backslash B) \geq \delta$ and $B \cap \mathbb{D}_{1 / 2} \neq \emptyset$, then $B \subset \mathbb{D}_{R}$ where $R=R(\delta)<11^{11}$ By the Koebe Distortion Theorem, $\psi^{-1}\left(\mathbb{D}_{R}\right) \subset$ $\mathbb{D}_{\kappa r}(x)$. If $\Delta$ intersects $\mathbb{D}_{r}(x)$ and $\partial \mathbb{D}_{\kappa r}(x)$, then $\psi(\Delta)$ intersects $\mathbb{D}_{1 / 2}$ and $\partial \mathbb{D}_{R}$, so that $\bmod (D \backslash \Delta)=\bmod (\mathbb{D} \backslash \psi(\Delta))<\delta$.

Given $\rho>0$, there exists $n$ such that $\mathcal{V}^{n} \subset \mathbb{D}_{\rho}(c), \bmod \left(\mathcal{V}^{n} \backslash \mathcal{V}^{n+1}\right)>\delta$ and $\bmod \left(V^{n} \backslash V^{n+1}\right)>\delta$. Then for some $\eta=\eta(\delta)$, we have $\bmod \left(\mathcal{V}^{n+1} \backslash \mathcal{V}^{n+2}(\tilde{c})\right)>\eta$ and

\footnotetext{
10 One can show that the parapuzzle pieces corresponding to the puzzle pieces in the enhanced nest constructed in [KSS| have a bounded shape, but this nest is less convenient for the statistical arguments.

11 The optimal choice is to take $R \in(1 / 2,1)$ such that $\bmod (\mathbb{D} \backslash[1 / 2, R])=\delta$.
} 
$\bmod \left(V^{n+1}(\tilde{c}) \backslash V^{n+2}(\tilde{c})\right)>\eta$ for every combinatorially recurrent parameter $\tilde{c} \in \mathcal{V}^{n+1}$. Almost every $\tilde{c} \in \mathcal{V}^{n+1} \cap \mathcal{M}$ is combinatorially recurrent, and by Corollary 5.4 and Theorem 6.3. $\mathbb{P}\left(\mathcal{M} \mid \mathcal{V}^{n+2}(\tilde{c})\right)<1-\epsilon$. By the previous lemma, there exists $r>0$ such that $\mathbb{D}_{r}(c) \subset \mathcal{V}^{n+1}$ and any $\mathcal{V}^{n+2}(\tilde{c})$ intersecting $\mathbb{D}_{r}(c)$ is contained $\mathbb{D}_{\kappa r}(c)$ for some $\kappa>1$. Let $X \subset \mathbb{D}_{\kappa r}(c)$ be the union of all the $\mathcal{V}^{n+2}(\tilde{c})$ intersecting $\mathbb{D}_{r}(c)$. Then

$$
1-\mathbb{P}\left(\mathcal{M} \mid \mathbb{D}_{\kappa r}(c)\right) \geq \kappa^{-2}(1-\mathbb{P}(\mathcal{M} \mid X)) \geq \epsilon \kappa^{-2} .
$$

Remark 6.1. Let us indicate how to generalize Theorem 1.4 to the finitely renormalizable case. We cannot just argue via renormalization since it would only prove that a finitely renormalizable parameter is not a density point of a copy of the Multibrot set containing it, and indeed a neighborhood of a satellite renormalizable parameter (with repelling periodic orbits) contains non-renormalizable parameters belonging to infinitely many Misiurewicz limbs.

This can be solved by constructing a different version of the puzzle and parapuzzle, which is designed to be compatible with a fixed renormalization. Namely, one constructs "adapted Yoccoz puzzle pieces of depth 0", where instead of using the external rays landing at the $\alpha$-fixed point of $f_{c}$, one uses the external rays landing at the orbit of the $\alpha$-fixed point of the renormalization of $f_{c}$. Though the combinatorial description is different (see [Sc2], [M3]), the whole geometric and statistical analysis can be carried out to obtain Theorem 1.4 in the more general setting.

Acknowledgments. We thank all the institutions and foundations that have supported us in the course of this work: Simons Mathematics and Physics Endowment, Fields Institute, NSF, NSERC, University of Toronto, Warwick Mathematics Institute. W.S. acknowledges support by the SRFDP grant No. 20070358058 and the 973 program grant No. 2006CB805900. This research was partially conducted during the period A.A. served as a Clay Research Fellow.

\section{References}

[ALM] Avila, A., Lyubich, M., de Melo, W.: Regular or stochastic dynamics in real analytic families of unimodal maps. Invent. Math. 154, 451-550 (2003) Zbl 1050.37018 MR 2018784

[AM1] Avila, A., Moreira, C. G.: Statistical properties of unimodal maps: the quadratic family. Ann. of Math. 161, 831-881 (2005) Zbl 1078.37029 MR 2153401

[AM2] Avila, A., Moreira, C. G.: Phase-parameter relation and sharp statistical properties for general families of unimodal maps. In: Geometry and Dynamics, Contemp. Math. 389, Amer. Math. Soc., 1-42 (2005) Zbl 1145.37022 MR 2181956

[AM3] Avila, A., Moreira, C. G.: Statistical properties of unimodal maps: physical measures, periodic orbits and pathological laminations. Publ. Math. IHÉS 101, 1-67 (2005) Zbl 1078.37030 MR 2217046

[AKLS] Avila, A., Kahn, J., Lyubich, M., Shen, W.: Combinatorial rigidity for unicritical polynomials. Ann. of Math. 170, 783-797 (2009) Zbl pre05610429 MR 2552107

[AL] Avila, A., Lyubich, M.: The full renormalization horseshoe for unimodal maps of higher degree: exponential contraction along hybrid classes. Preprint IMS at Stony Brook, \#3 (2010) 
[BC] Benedicks, M., Carleson, L.: On iterations of $1-a x^{2}$ on $(-1,1)$. Ann. of Math. 122, 1-25 (1985) Zbl 0597.58016 MR 0799250

[BR] Bers, L., Royden, H. L.: Holomorphic families of injections. Acta Math. 157, 259-286 (1986) Zbl 0619.30027 MR 0857675

[BKNS] Bruin, H., Keller, G., Nowicki, T., van Strien, S.: Wild Cantor attractors exist. Ann. of Math. (2) 143, 97-130 (1996) Zbl 0848.58016 MR 1370759

[BSS1] Bruin, H., Shen, W., van Strien, S.: Invariant measures exist without a growth condition. Comm. Math. Phys. 241, 287-306 (2003) Zbl 1098.37034 MR 2013801

[BSS2] Bruin, H., Shen, W., van Strien, S.: Existence of unique SRB-measures is typical for real unicritical polynomial families. Ann. Sci. École Norm. Sup. (4) 39, 381-414 (2006) Zbl 1112.37018 MR 2265674

[DH1] Douady, A., Hubbard, J. H.: Étude dynamiques des polynômes complexes. Publ. Math. d'Orsay, 84-02 (1984) and 85-04 (1985) Zbl 0571.30026 and Zbl 0552.30018. MR 0762431 and MR 0812271

[DH2] Douady, A., Hubbard, J. H.: On the dynamics of polynomial-like maps. Ann. Sci. École Norm. Sup. 18, 287-344 (1985) Zbl 0587.30028 MR 0816367

[GM] Goldberg, L. R., Milnor, J.: Fixed points of polynomial maps. II. Fixed point portraits. Ann. Sci. École Norm. Sup. (4) 26, 51-98 (1993) Zbl 0771.30028 MR 1209913

$[\mathrm{H}] \quad$ Hubbard, J. H.: Local connectivity of Julia sets and bifurcation loci: three theorems of J.-C. Yoccoz. In: Topological Methods in Modern Mathematics (Stony Brook, NY, 1991), Publish or Perish, Houston, TX, 467-511 (1993) Zbl 0797.58049 MR 1215974

[J] Jakobson, M.: Absolutely continuous invariant measures for one-parameter families of one-dimensional maps. Comm. Math. Phys. 81, 39-88 (1981) Zbl 0497.58017 MR 0630331

[K] Kahn, J.: Holomorphic removability of Julia sets. Preprint IMS at Stony Brook, \# 11 (1998)

[KL1] Kahn, J., Lyubich, M.: The quasi-additivity law in conformal geometry. Ann. of Math. 169, 561-593 (2009) Zbl pre05578751 MR 2480612

[KL2] Kahn, J., Lyubich, M.: Local connectivity of Julia sets for unicritical polynomials. Ann. of Math. 170, 413-426 (2009) Zbl pre05578965 MR 2521120

[KSS] Kozlovski, O., Shen, W., van Strien, S.: Rigidity for real polynomials. Ann. of Math. (2) 165, 749-841 (2007) Zbl 1129.37020 MR 2335796

[L1] Lyubich, M.: On the Lebesgue measure of the Julia set of a quadratic polynomial. Preprint IMS at Stony Brook, \# 10 (1991)

[L2] Lyubich, M.: Combinatorics, geometry and attractors of quasi-quadratic maps. Ann. of Math. 140, 347-404 (1994) Zbl 0821.58014 MR 1298717

[L3] Lyubich, M.: Dynamics of quadratic polynomials. I, II. Acta Math. 178, 185-247, 247297 (1997) Zbl 0908.58053 MR 1459261

[L4] Lyubich, M.: Dynamics of quadratic polynomials, III. Parapuzzle and SBR measure. Astérisque 261, 173-200 (2000) Zbl 1044.37038 MR 1755441

[L5] Lyubich, M.: Almost every real quadratic maps is either regular or stochastic. Ann. of Math. 156, 1-78 (2002) Zbl 1160.37356 MR 1935840

[MSS] Mañé, R., Sad, P., Sullivan, D.: On the dynamics of rational maps, Ann. Sci. École Norm. Sup. 16, 193-217 (1983) Zbl 0524.58025 MR 0732343

[MN] Martens, M., Nowicki, T.: Invariant measures for Lebesgue typical quadratic maps. Astérisque 261, 239-252 (2000) Zbl 0939.37020 MR 1755443

[M1] Milnor, J.: On the concept of attractor. Comm. Math. Phys. 99, 177-195 (1985) Zbl 0595.58028 MR 0790735 
[M2] Milnor, J.: Local connectivity of Julia sets: Expository lectures. In: The Mandelbrot Set: Themes and Variations, London Math. Soc. Lecture Note Ser. 274, Tan Lei (ed.), Cambridge Univ. Press, 67-116 (2000) Zbl 1107.37305 MR 1765085

[M3] Milnor, J.: Periodic orbits, external rays, and the Mandelbrot set: Expository account. In: Géométrie Complexe et Systèmes Dynamiques, Astérisque 261, 277-333 (2000) Zbl 0941.30016 MR 1755445

[RL] Rivera-Letelier, J.: On the continuity of Hausdorff dimension of Julia sets and similarity between the Mandelbrot set and Julia sets. Fund. Math. 170, 287-317 (2001) Zbl 0985.37041 MR 1880905

[Sa] Sands, D.: Misiurewicz maps are rare. Comm. Math. Phys. 197, 109-129 (1998) Zbl 0921.58015 MR 1646471

[Sc1] Schleicher, D.: Rational parameter rays of the Mandelbrot set. Astérisque 261, 405-443 (2000) Zbl 0941.30015 MR 1755449

[Sc2] Schleicher, D.: On fibers and local connectivity of Mandelbrot and Multibrot sets. In: Fractal Geometry and Applications: a jubilee of Benoît Mandelbrot, Proc. Sympos. Pure Math. 72, Part 1, Amer. Math. Soc., Providence, RI, 477-517 (2004) Zbl 1074.30025 MR 2112117

[S] Shishikura, M.: Topological, geometric and complex analytic properties of Julia sets. In: Proc. International Congress of Mathematicians (Zürich, 1994), Birkhäuser, Basel, 886895 (1995) Zbl 0843.30026 MR 1403988

[S1] Słodkowski, Z.: Holomorphic motions and polynomial hulls. Proc. Amer. Math. Soc. 111, 347-355 (1991)

[Sm] Smania, D.: Puzzle geometry and rigidity: The Fibonacci cycle is hyperbolic. J. Amer. Math. Soc. 20, 629-673 (2007) Zbl pre05144251 MR 2291915

[vS] van Strien, S.: Misiurewicz points unfold generically (even if they are critically nonfinite). Fund. Math. 163, 39-54 (2000) Zbl 0965.37038 MR 1750334

[T] Tsujii, M.: Positive Lyapunov exponents in families of one dimensional dynamical systems. Invent. Math. 111, 113-137 (1993) Zbl 0787.58029 MR 1193600 